\title{
Existence and Uniqueness of Solution of a Continuous Flow Bioreactor Model with Two Species.
}

\author{
M. CRESPO ${ }^{1, a}$, B. IVORRA ${ }^{1, b}$ AND A.M RAMOS ${ }^{1, c}$ \\ ${ }^{1}$ Departamento de Matemática Aplicada \& Instituto de Matemática Interisciplinar, \\ Universidad Complutense de Madrid. Plaza de Ciencias, 3, 28040 Madrid, Spain. \\ a Tel.: +34-913944462. E-mail: mcresp01@ucm.es \\ b Tel.: +34-913944415. E-mail: ivorra@mat.ucm.es \\ c Tel.: +34-913944480. E-mail: angel@mat.ucm.es
}

\begin{abstract}
In this work, we study the mathematical analysis of a coupled system of two reactiondiffusion-advection equations and Danckwerts boundary conditions, which models the interaction between a microbial population (e.g., bacterias) and a diluted substrate (e.g., nitrate) in a continuous flow bioreactor. This type of bioreactor can be used, for instance, for water treatment. First, we prove the existence and uniqueness of solution, under the hypothesis of linear reaction by using classical results for linear parabolic boundary value problems. Next, we prove the existence and uniqueness of solution for some nonlinear reactions by applying Schauder Fixed Point Theorem and the theorem obtained for the linear case. Results about the nonnegativeness and boundedness of the solution are also proved here.
\end{abstract}

\section{Introduction}

Water treatment is an important environmental issue whose main objective is to provide clean water to human populations (see, e.g., 2 2). One of the principal causes of contamination of water resources is due to organic or mineral substrates (e.g., nitrates or phosphorus) which are produced by the agriculture and chemical sectors. A way to perform the decontamination of these substrates is to use a bioreactor. In our framework, a bioreactor is a vessel in which a microorganism (e.g., bacteria or yeast), called biomass, is used to degrade a considered diluted substrate. Developing mathematical models that allow to simulate the interaction between biomass and substrate inside a bioreactor is of great interest in order to design efficient water treatment devices (see, e.g., [5, 11]).

There exists many mathematical models describing the competition between biomass and substrate in bioreactors. Most theoretical studies consider a well-mixed environment, such as the chemostat (see, e.g., [26]). Focusing on bacterias, some of the first explorations of bacterial growth in spatially distributed environments, were carried out by Lauffenburger, Aris and Keller [14] and Lauffenburger and Calcagno [15. Particularly, Kung and Baltzis [12] considered a tubular bioreactor(assumed to be a thin tube), through which a liquid charged with a substrate at constant concentration enters the bioreactor with a constant flow rate, and the outflow leaves the bioreactor with the same flow rate. These considerations lead to a coupled system of two reaction-diffusion-advection equations with Danckwerts boundary conditions, typically used for continuous flows bioreactors (see, e.g., [12, 28, 3]). 
This system of parabolic equations has received considerable attention in the literature, both from theoretical and applied points of view. One can find the one-dimensional version of the model with Danckwerts boundary conditions in [4], [7] and [22], where the asymptotic behavior of the solution is studied under the assumption of constant fluid flow and entering substrate. There exist many works on the existence and uniqueness of solution of linear parabolic equations [1] 8] [9] [13, particularly, for general bounded domains (see, e.g., [18, 19, 17, 16]). For the existence and uniqueness of solution of nonlinear parabolic systems in $\mathcal{C}^{1+\alpha}$ domains with mixed boundary conditions one can see the work developed by Pao [20, [21], where the method of lower and upper solutions is used. The existence and uniqueness for a predator-prey type model with nonlinear reaction term is proved in [25] for Neumann boundary conditions.

In this work, we carry out a mathematical analysis of a coupled system of two reactiondiffusion-advection equations completed with Danckwerts boundary conditions, which models the interaction between a substrate and a biomass, whose concentrations are denoted by $S$ and $B$, respectively. We prove the existence and uniqueness of (weak) solutions, together with results about the nonnegativeness and boundedness of the solution. The reaction term is assumed nonlinear in $S$. The domain into consideration is a three-dimensional cylindrical bioreactor with Lipschitz boundary. The bioreactor is fed with a substrate concentration $S_{\mathrm{e}}$ at flow rate $Q$, and the treated outflow leaves the bioreactor with the same flow rate $Q$. In contrast to the models presented in [4, 7] and [22, we allow variable $Q$ to vary with time and space, we also allow $S_{\mathrm{e}}$ to vary with time and we consider a three-dimensional domain with Lipschitz boundary.

This papers is organized as follows: Section 2 introduces the mathematical model which describes the behavior of the continuous flow bioreactor and considers nonlinear reaction between the biomass and the substrate. We also state the definition of weak solution. In Section 3, we first prove the existence and uniqueness of solution of a simplified linear system through some classical results for linear parabolic systems boundary value problems. Then, we prove the existence and uniqueness of solution of the nonlinear system applying the Schauder Fixed Point Theorem.

\section{Mathematical modelling and weak solutions}

We consider a cylindrical bioreactor as the one showed in Figure 1 . We denote by $\Omega \subset \mathbb{R}^{3}$ its spatial domain, by $\delta \Omega=\Gamma$ its boundary and by $\bar{\Omega}$ their union, i.e, $\bar{\Omega}=\Omega \cup \delta \Omega$. We assume that $\Gamma=\Gamma_{\text {in }} \cup \Gamma_{\text {out }} \cup \Gamma_{\text {wall }}$, where $\Gamma_{\text {in }}$ is the inlet upper boundary, $\Gamma_{\text {out }}$ is the outlet lower boundary and $\Gamma_{\text {wall }}$ corresponds to the bioreactor lateral walls. At the beginning of the process, there is a certain amount of biomass and substrate inside $\Omega$. Furthermore, during the studied time interval, diluted substrate enters the device through the inlet $\Gamma_{\text {in }}$ and the fluid exits the bioreactor through the outlet $\Gamma_{\text {out }}$. We consider the following system describing the 


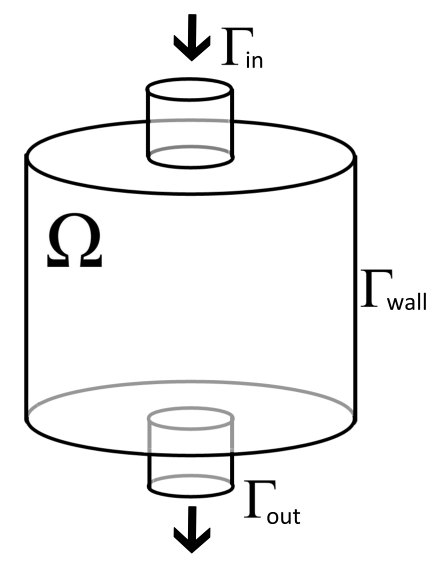

Figure 1: Typical domain representation of the bioreactor geometry.

behavior of this particular bioreactor

$$
\left\{\begin{array}{lr}
S_{t}=\operatorname{div}\left(D_{\mathrm{S}} \nabla S-\mathbf{Q} S\right)-\mu(S) B & \text { in } \Omega \times(0, T), \\
B_{t}=\operatorname{div}\left(D_{\mathrm{B}} \nabla B-\mathbf{Q} B\right)+\mu(S) B & \text { in } \Omega \times(0, T), \\
S(x, 0)=S_{\text {init }}(x) & \text { in } \Omega, \\
B(x, 0)=B_{\text {init }}(x) & \text { in } \Omega, \\
\mathbf{n} \cdot\left(D_{\mathrm{S}} \nabla S-\mathbf{Q} S\right)=S_{\mathrm{e}} Q & \text { in } \Gamma_{\text {in }} \times(0, T), \\
\mathbf{n} \cdot\left(D_{\mathrm{B}} \nabla B-\mathbf{Q} B\right)=0 & \Gamma_{\text {in }} \cup \Gamma_{\text {wall }} \times(0, T), \\
\mathbf{n} \cdot\left(D_{\mathrm{S}} \nabla S-\mathbf{Q} S\right)=0 & \text { in } \Gamma_{\text {wall }} \times(0, T), \\
\mathbf{n} \cdot\left(D_{\mathrm{S}} \nabla S\right)=0 & \text { in } \Gamma_{\text {out }} \times(0, T), \\
\mathbf{n} \cdot\left(D_{\mathrm{B}} \nabla B\right)=0 & \text { in } \Gamma_{\text {out }} \times(0, T),
\end{array}\right.
$$

where $T>0$ (s) is the length of the time interval for which we want to model the process, $S$ $\left(\mathrm{mol} / \mathrm{m}^{3}\right)$ and $B\left(\mathrm{~mol} / \mathrm{m}^{3}\right)$ are the substrate and biomass concentration inside the bioreactor, which diffuse throughout the water in the vessel with diffusion coefficients $D_{\mathrm{S}}\left(\mathrm{m}^{2} / \mathrm{s}\right), D_{\mathrm{B}}$ $\left(\mathrm{m}^{2} / \mathrm{s}\right)$, respectively. The fluid flow is taken as $\mathbf{Q}=(0,0,-Q(x, t))$ where $Q(\mathrm{~m} / \mathrm{s})$ is the flow rate. $S_{\mathrm{e}}(t)\left(\mathrm{mol} / \mathrm{m}^{3}\right)$ is the concentration of substrate that enters into the bioreactor at time $t(\mathrm{~s}), S_{\text {init }}\left(\mathrm{mol} / \mathrm{m}^{3}\right)$ and $B_{\text {init }}\left(\mathrm{mol} / \mathrm{m}^{3}\right)$ are the concentration of substrate and biomass inside the bioreactor at the beginning of the process, respectively, and $\mathbf{n}$ is the outward unit normal vector on the boundary of the domain $\Omega$. Notice that besides the Advection-Diffusion terms, we also have a term corresponding to the reaction of biomass and substrate, governed by the growth rate function $\mu(\cdot)\left(\mathrm{s}^{-1}\right)$.

Now, we are interested in defining the concept weak solutions for our System 1, To do so, assuming $S, B \in W\left(0, T, H^{1}(\Omega),\left(H^{1}(\Omega)\right)^{\prime}\right)$ (see the definition of this set in Appendix [A.1), $Q \in L^{\infty}(0, T, \mathcal{C}(\bar{\Omega})), S_{\mathrm{e}} \in L^{2}(0, T)$ and $\mu \in L^{\infty}(\mathbb{R})$, if we multiply the first equation of (1) by 
$v \in H^{1}(\Omega)$, it follows that

$$
\begin{aligned}
<S_{t}, v & >_{\left(H^{1}(\Omega)\right)^{\prime} \times H^{1}(\Omega)}-<\operatorname{div}\left(D_{\mathrm{S}} \nabla S-\mathbf{Q} S\right), v>_{\left(H^{1}(\Omega)\right)^{\prime} \times H^{1}(\Omega)} \\
& +\int_{\Omega} \mu(S(x, t)) B(x, t) v(x) \mathrm{d} x=0 .
\end{aligned}
$$

Then, applying the Green's Formula and taking into account the boundary conditions, we obtain

$<S_{t}, v>_{\left(H^{1}(\Omega)\right)^{\prime} \times H^{1}(\Omega)}+\int_{\Omega} \mu(S(x, t)) B(x, t) v(x) \mathrm{d} x-\int_{\Gamma_{\mathrm{in}}} Q(x, t) S_{\mathrm{e}}(t) v(x) \mathrm{d} \Gamma_{\mathrm{in}}$

$+\int_{\Omega}\left(D_{\mathrm{S}} \nabla S(x, t)-\mathbf{Q}(x, t) S(x, t)\right) \nabla v(x) \mathrm{d} x+\int_{\Gamma_{\text {out }}} Q(x, t) S(x, t) v(x) \mathrm{d} \Gamma_{\text {out }}=0$.

Similarly, multiplying the second equation of (10) by $w \in H^{1}(\Omega)$, applying the Green's Formula and taking into account the boundary conditions, one has that

$<B_{t}, w>_{\left(H^{1}(\Omega)\right)^{\prime} \times H^{1}(\Omega)}+\int_{\Omega}\left(D_{\mathrm{B}} \nabla B(x, t)-\mathbf{Q}(x, t) B(x, t)\right) \nabla w(x) \mathrm{d} x$

$$
+\int_{\Gamma_{\text {out }}} Q(x, t) B(x, t) w(x) \mathrm{d} \Gamma_{\text {out }}-\int_{\Omega} \mu(S(x, t)) B(x, t) w(x) \mathrm{d} x=0 .
$$

We define the operators $a_{1}:(0, T) \times H^{1}(\Omega) \times H^{1}(\Omega) \rightarrow \mathbb{R}$ and $a_{2}:(0, T) \times H^{1}(\Omega) \times H^{1}(\Omega)$ by

$$
\begin{aligned}
a_{1}(t, p, v) & =\int_{\Omega}\left(D_{\mathrm{S}} \nabla p(x)-\mathbf{Q}(x, t) p(x)\right) \nabla v(x) \mathrm{d} x+\int_{\Gamma_{\text {out }}} Q(x, t) p(x) v(x) \mathrm{d} \Gamma_{\text {out }}, \\
a_{2}(t, q, w) & =\int_{\Omega}\left(D_{\mathrm{B}} \nabla q(x)-\mathbf{Q}(x, t) q(x)\right) \nabla w(x) \mathrm{d} x+\int_{\Gamma_{\text {out }}} Q(x, t) q(x) w(x) \mathrm{d} \Gamma_{\text {out }} .
\end{aligned}
$$

Let us denote $\boldsymbol{\psi}=\left(\begin{array}{c}p \\ q\end{array}\right), \boldsymbol{\phi}=\left(\begin{array}{c}v \\ w\end{array}\right), \mathbf{H}^{1}(\Omega)=H^{1}(\Omega) \times H^{1}(\Omega)$ and $\left(\mathbf{H}^{1}(\Omega)\right)^{\prime}=\left(H^{1}(\Omega)\right)^{\prime} \times$ $\left(H^{1}(\Omega)\right)^{\prime}$ and consider the bilinear form

$A(t, \cdot, \cdot): \mathbf{H}^{1} \times \mathbf{H}^{1} \rightarrow \mathbb{R}$ defined by:

$$
A(t, \boldsymbol{\psi}, \boldsymbol{\phi})=a_{1}(t, p, v)+a_{2}(t, q, w) .
$$

Definition 2.1. A weak solution of problem (1) is a function $\mathbf{u}=(S, B)$ such that $S, B \in$ $W\left(0, T, H^{1}(\Omega),\left(H^{1}(\Omega)\right)^{\prime}\right)$ and satisfy

$$
\left\{\begin{array}{r}
<\mathbf{u}_{t}(\cdot), \boldsymbol{\phi}>_{\left(\mathbf{H}^{1}(\Omega)\right)^{\prime} \times \mathbf{H}^{1}(\Omega)}+A(\cdot, \mathbf{u}(\cdot), \boldsymbol{\phi})= \\
\quad \int_{\Gamma_{\mathrm{in}}} Q(x, \cdot) S_{\mathrm{e}}(\cdot) v(x) \mathrm{d} \Gamma_{\mathrm{in}}+\int_{\Omega} \mu(S(x, \cdot)) B(x, \cdot)(w(x)-v(x)) \mathrm{d} x \\
\quad \text { for all } \boldsymbol{\phi}=(v, w) \in \mathbf{H}^{1}(\Omega)
\end{array}\right.
$$

in the sense of $\mathcal{D}^{\prime}(0, T)$ (see, e.g., [10]), i.e., all the terms above are considered as distributions in $t$. Notice that

$$
\begin{aligned}
<\mathbf{u}_{t}(\cdot), \phi>_{\left(\mathbf{H}^{1}(\Omega)\right)^{\prime} \times \mathbf{H}^{1}(\Omega)} & =<S_{t}(\cdot)+B_{t}(\cdot), v>_{\left(H^{1}(\Omega)\right)^{\prime} \times H^{1}(\Omega)} \\
& =\frac{\mathrm{d}}{\mathrm{d} t}\left(\int_{\Omega} S(\cdot, x) v(x) \mathrm{d} x+\int_{\Omega} B(\cdot, x) w(x) \mathrm{d} x\right) \text { in the sense of } \mathcal{D}^{\prime}(0 . T) .
\end{aligned}
$$




\section{Existence, uniqueness, nonnegativity and boundedness of the solution}

We are first interested in proving the following result:

Theorem 3.1 (Existence of solution). Let us assume that $Q \in L^{\infty}(0, T, \mathcal{C}(\bar{C}))$ is nonnegative, $S_{\mathrm{e}} \in L^{2}(0, T), S_{\text {init }}, B_{\text {init }} \in L^{2}(\Omega), D_{\mathrm{S}}, D_{\mathrm{B}}>0$ and $\mu \in L^{\infty}(\mathbb{R})$ is continuous. Then, System (1) has at least one weak solution $(S, B)$.

Remark 3.2. Notice that we assume that $Q$ is nonnegative because of its physical meaning. However, in order to prove Theorem 3.1 it suffices to consider $Q$ such that $\|Q(\cdot, t)\|_{L^{\infty}(\bar{\Omega})}<$ $\frac{\min \left(D_{\mathrm{S}}, D_{\mathrm{B}}\right)}{C_{\mathrm{T}}^{2}}$ a.e. $t \in(0, T)$, where $C_{\mathrm{T}}$ is the constant coming from the Trace Theorem (see A.9).

In order to prove Theorem 3.1, we first investigate the existence and uniqueness of solution of the following linear parabolic system:

$$
\left\{\begin{array}{lr}
S_{t}-\operatorname{div}\left(D_{\mathrm{S}} \nabla S-\mathbf{Q} S\right)+c B=0 & \text { in } \Omega \times(0, T), \\
B_{t}-\operatorname{div}\left(D_{\mathrm{B}} \nabla B-\mathbf{Q} B\right)-c B=0 & \text { in } \Omega \times(0, T), \\
S(x, 0)=S_{\text {init }}(x) & \text { in } \Omega, \\
B(x, 0)=B_{\text {init }}(x) & \text { in } \Omega, \\
\mathbf{n} \cdot\left(D_{\mathrm{S}} \nabla S-\mathbf{Q} S\right)=S_{\mathrm{e}} Q & \text { in } \Gamma_{\text {in }} \times(0, T), \\
\mathbf{n} \cdot\left(D_{\mathrm{B}} \nabla B-\mathbf{Q} B\right)=0 & \Gamma_{\text {in }} \cup \Gamma_{\text {wall }} \times(0, T), \\
\mathbf{n} \cdot\left(D_{\mathrm{S}} \nabla S-\mathbf{Q} S\right)=0 & \text { in } \Gamma_{\text {wall }} \times(0, T), \\
\mathbf{n} \cdot\left(D_{\mathrm{S}} \nabla S\right)=0 & \text { in } \Gamma_{\text {out }} \times(0, T), \\
\mathbf{n} \cdot\left(D_{\mathrm{B}} \nabla B\right)=0 & \text { in } \Gamma_{\text {out }} \times(0, T),
\end{array}\right.
$$

where $c \in L^{\infty}(\Omega \times(0, T))$.

Analogously to the nonlinear case, we first define the concept of weak solution for this system. To do so, if we multiply the first equation of (3) by $v \in H^{1}(\Omega)$, it follows that

$<S_{t}, v>_{\left(H^{1}(\Omega)\right)^{\prime} \times H^{1}(\Omega)}-\int_{\Gamma_{\mathrm{in}}} Q(x, t) S_{\mathrm{e}}(t) v(x) \mathrm{d} \Gamma_{\mathrm{in}}+\int_{\Omega} c(x, t) B(x, t) v(x) \mathrm{d} x$

$+\int_{\Omega}\left(D_{\mathrm{S}} \nabla S(x, t)-\mathbf{Q}(x, t) S(x, t)\right) \nabla v(x) \mathrm{d} x+\int_{\Gamma_{\text {out }}} Q(x, t) S(x, t) v(x) \mathrm{d} \Gamma_{\text {out }}=0$.

Similarly, if we multiply the second equation of (3) by $w \in H^{1}(\Omega)$, we obtain that

$<B_{t}, w>_{\left(H^{1}(\Omega)\right)^{\prime} \times H^{1}(\Omega)}+\int_{\Gamma_{\text {out }}} Q(x, t) B(x, t) w(x) \mathrm{d} \Gamma_{\text {out }}$

$$
+\int_{\Omega}\left(D_{\mathrm{B}} \nabla B(x, t)-\mathbf{Q}(x, t) B(x, t)\right) \nabla w(x) \mathrm{d} x-\int_{\Omega} c(x, t) B(x, t) w(x) \mathrm{d} x=0 .
$$

We define the operators $\bar{a}_{1}:(0, T) \times \mathbf{H}^{1}(\Omega) \times H^{1}(\Omega)$ and $\bar{a}_{2}:(0, T) \times H^{1}(\Omega) \times H^{1}(\Omega)$ 
by

$$
\begin{aligned}
\bar{a}_{1}(t,(p, q), v)= & \int_{\Omega}\left(D_{\mathrm{S}} \nabla p(x)-\mathbf{Q}(x, t) p(x)\right) \nabla v(x) \mathrm{d} x \\
& +\int_{\Omega} c(x, t) q(x) v(x) \mathrm{d} x+\int_{\Gamma_{\text {out }}} Q(x, t) p(x) v(x) \mathrm{d} \Gamma_{\text {out }}, \\
\bar{a}_{2}(t, q, w)= & \int_{\Omega}\left(D_{\mathrm{B}} \nabla q(x)-\mathbf{Q}(x, t) q(x)\right) \nabla w(x) \mathrm{d} x-\int_{\Omega} c(x, t) q(x) w(x) \mathrm{d} x \\
& +\int_{\Gamma_{\text {out }}} Q(x, t) q(x) w(x) \mathrm{d} \Gamma_{\text {out }} .
\end{aligned}
$$

Let us denote $\boldsymbol{\psi}=\left(\begin{array}{c}p \\ q\end{array}\right), \boldsymbol{\phi}=\left(\begin{array}{c}v \\ w\end{array}\right)$ and consider the bilinear form $\bar{A}(t, \cdot, \cdot): \mathbf{H}^{1} \times \mathbf{H}^{1} \rightarrow \mathbb{R}$ defined by

$$
\bar{A}(t, \boldsymbol{\psi}, \boldsymbol{\phi})=\bar{a}_{1}(t,(p, q), v)+\bar{a}_{2}(t, q, w) .
$$

Definition 3.3. A weak solution of problem (3) is a function $\mathbf{u}=(S, B)$ such that $S, B \in W\left(0, T, H^{1}(\Omega),\left(H^{1}(\Omega)\right)^{\prime}\right)$ and satisfy

$$
\left\{\begin{array}{c}
\left\langle\mathbf{u}_{t}(\cdot), \phi>_{\left(\mathbf{H}^{1}(\Omega)\right)^{\prime} \times \mathbf{H}^{1}(\Omega)}+\bar{A}(\cdot, \mathbf{u}(\cdot), \phi)=\int_{\Gamma_{\mathrm{in}}} Q(x, \cdot) S_{\mathrm{e}}(\cdot) v(x) \mathrm{d} \Gamma_{\mathrm{in}}\right. \\
\text { for all } \left.\phi=(v, w) \in \mathbf{H}^{1}(\Omega)\right)
\end{array}\right.
$$

in the sense of $\mathcal{D}^{\prime}(0, T)$.

We now focus on proving the existence and uniqueness of solution of the linear system:

Theorem 3.4. Under the assumptions of Theorem [3.1, problem (3) has a unique weak solution $(S, B)$.

Proof of Theorem 3.4. In order to prove Theorem 3.4, we use Theorem A.3. Therefore, we need to choose suitable Hilbert spaces $V$ and $H$ such that $V \subset H$ and $V$ is dense in $H$.

We consider $V=\mathbf{H}^{1}(\Omega)=H^{1}(\Omega) \times H^{1}(\Omega)$ and $H=\mathbf{L}^{2}(\Omega)=L^{2}(\Omega) \times L^{2}(\Omega)$, provided with the norms

$$
\|\boldsymbol{\psi}\|_{\mathbf{H}^{1}(\Omega)}^{2}=\|p\|_{\mathbf{H}^{1}(\Omega)}^{2}+\|q\|_{\mathbf{H}^{1}(\Omega)}^{2} \quad \text { where } \boldsymbol{\psi}=(p, q) \in \mathbf{H}^{1}(\Omega),
$$

and

$$
\|p\|_{H^{1}(\Omega)}^{2}=\|p\|_{L^{2}(\Omega)}^{2}+\|\nabla p\|_{L^{2}(\Omega)}^{2} \quad \text { for any } p \in H^{1}(\Omega) .
$$

Let us see that $\bar{A}$ satisfies condition (33):

For all $\boldsymbol{\psi}, \boldsymbol{\phi}, \in \mathbf{H}^{1}(\Omega)$, function $t \rightarrow \bar{A}(t, \boldsymbol{\psi}, \boldsymbol{\phi})$ is Lebesgue measurable. This follows from the fact that $c$ and $Q$ are assumed to be Lebesgue measurable functions.

To be able to apply Theorem A.3, we need to find $k \in \mathbb{R}$ such that $|\bar{A}(t, \boldsymbol{\psi}, \boldsymbol{\phi})| \leq$ $k\|\boldsymbol{\psi}\|_{\mathbf{H}^{1}(\Omega)}\|\boldsymbol{\phi}\|_{\mathbf{H}^{1}(\Omega)}$ for all $\boldsymbol{\psi}, \boldsymbol{\phi} \in \mathbf{H}^{1}(\Omega)$, a.e.t $\in(0, T)$.

Now,

$$
\begin{aligned}
\left|\bar{a}_{1}(t,(p, q), v)\right|=\mid \int_{\Omega} D_{\mathrm{S}} \nabla p(x) \nabla v(x) \mathrm{d} x-\int_{\Omega} \mathbf{Q}(x, t) p(x) \nabla p(x) \mathrm{d} x \\
\quad+\int_{\Omega} c(x, t) q(x) v(x) \mathrm{d} x+\int_{\Gamma_{\text {out }}} Q(x, t) p(x) v(x) \mathrm{d} \Gamma_{\text {out }} \mid
\end{aligned}
$$

$\leq D_{\mathrm{S}} \int_{\Omega}|\nabla p(x)||\nabla v(x)| \mathrm{d} x+\|Q\|_{L^{\infty}(\bar{\Omega} \times(0, T))} \int_{\Omega}|p(x)||\nabla v(x)| \mathrm{d} x$ 


$$
+\|c\|_{L^{\infty}(\Omega \times(0, T))}\|q\|_{L^{2}(\Omega)}\|v\|_{L^{2}(\Omega)}+\|Q\|_{L^{\infty}(\bar{\Omega} \times(0, T))}\|p\|_{L^{2}\left(\Gamma_{\text {out }}\right)}\|v\|_{L^{2}\left(\Gamma_{\text {out }}\right)} .
$$

Then, using the Trace Theorem A.9, we can conclude that there exist a constant $C_{\mathrm{T}}>0$ such that

$$
\begin{aligned}
\left|\bar{a}_{1}(t,(p, q), v)\right| \leq & \underbrace{\left(D_{\mathrm{S}}+\left(1+C_{\mathrm{T}}^{2}\right)\|Q\|_{L^{\infty}(\bar{\Omega} \times(0, T))}\right.}_{\left(k_{1}\right)}\|p\|_{H^{1}(\Omega)}\|v\|_{H^{1}(\Omega)} \\
& +\underbrace{\|c\|_{L^{\infty}(\Omega \times(0, T))}}_{\left(k_{2}\right)}\|q\|_{H^{1}(\Omega)}\|v\|_{H^{1}(\Omega)} \\
\leq & \left(k_{1}+k_{2}\right)\|(p, q)\|_{\mathbf{H}^{1}(\Omega)}\|(v, w)\|_{\mathbf{H}^{1}(\Omega)}=\left(k_{1}+k_{2}\right)\|\boldsymbol{\psi}\|_{\mathbf{H}^{1}(\Omega)}\|\boldsymbol{\phi}\|_{\mathbf{H}^{1}(\Omega)} .
\end{aligned}
$$

Similarly, we obtain that

$$
\begin{aligned}
& \left|\bar{a}_{2}(t, q, w)\right|=\mid \int_{\Omega} D_{\mathrm{B}} \nabla q(x) \nabla w(x) \mathrm{d} x-\int_{\Omega} \mathbf{Q}(x, t) q(x) w(x) \mathrm{d} x \\
& -\int_{\Omega} c(x, t) q(x) w(x) \mathrm{d} x+\int_{\Gamma_{\text {out }}} Q(x, t) q(x) w(x) \mathrm{d} \Gamma_{\text {out }} \mid \\
& \leq \underbrace{\left(D_{\mathrm{B}}+\left(1+C_{\mathrm{T}}^{2}\right)\|Q\|_{L^{\infty}(\bar{\Omega} \times(0, T))}+\|c\|_{L^{\infty}(\Omega \times(0, T))}\right.}_{\left(k_{3}\right)}\|q\|_{H^{1}(\Omega)}\|w\|_{H^{1}(\Omega)}
\end{aligned}
$$

$\leq k_{3}\|(p, q)\|_{\mathbf{H}^{1}(\Omega)}\|(v, w)\|_{\mathbf{H}^{1}(\Omega)}=k_{3}\|\boldsymbol{\psi}\|_{\mathbf{H}^{1}(\Omega)}\|\boldsymbol{\phi}\|_{\mathbf{H}^{1}(\Omega)}$.

Consequently, it follows

$$
|\bar{A}(t, \boldsymbol{\psi}, \boldsymbol{\phi})| \leq \underbrace{\left(k_{1}+k_{2}+k_{3}\right)}_{(k)}\|\boldsymbol{\psi}\|_{\mathbf{H}^{1}(\Omega)}\|\boldsymbol{\phi}\|_{\mathbf{H}^{1}(\Omega)} .
$$

Let us see that $\bar{A}$ satisfies condition (34):

We need to find $\alpha, \lambda>0$ such that $\bar{A}(t,(p, q),(p, q))+\lambda\left(\|p\|_{L^{2}}^{2}+\|q\|_{L^{2}}^{2}\right) \geq \alpha\|(p, q)\|_{\mathbf{H}^{1}(\Omega)}^{2}$ for all $p, q \in H^{1}(\Omega)$, a.e. $t \in(0, T)$, taking into account that $\bar{A}(t,(p, q),(p, q))=\bar{a}_{1}(t,(p, q), p)+$ $\bar{a}_{2}(t, q, q)$. We have that

$$
\begin{aligned}
\bar{a}_{1}(t,(p, q), p)= & D_{\mathrm{S}}\|\nabla p\|_{L^{2}(\Omega)}^{2}-\int_{\Omega} \mathbf{Q}(x, t) p(x) \nabla p(x) \mathrm{d} x+\int_{\Omega} c(x, t) q(x) p(x) \mathrm{d} x \\
& +\int_{\Gamma_{\text {out }}} Q(x, t) p(x)^{2} \mathrm{~d} x,
\end{aligned}
$$

and

$$
\begin{aligned}
\bar{a}_{2}(t, q, q)= & D_{\mathrm{B}}\|\nabla q\|_{L^{2}(\Omega)}^{2}-\int_{\Omega} \mathbf{Q}(x, t) q(x) \nabla q(x) \mathrm{d} x+\int_{\Omega} c(x, t) q^{2}(x) \mathrm{d} x \\
& +\int_{\Gamma_{\text {out }}} Q(x, t) q(x)^{2} \mathrm{~d} x .
\end{aligned}
$$

Applying Young's inequality (36) with $\epsilon>0$, to be chosen later, and taking $z=p$ or $z=q$ the following inequality holds:

$-\int_{\Omega} \mathbf{Q}(x, t) z(x) \nabla z(x) \mathrm{d} x \geq-\left(\epsilon\|z\|_{L^{2}(\Omega)}^{2}+\frac{1}{4 \epsilon}\|\nabla z\|_{L^{2}(\Omega)}^{2}\right)\|Q\|_{L^{\infty}(\bar{\Omega} \times(0, T))}$.

Furthermore, 
$\int_{\Gamma_{\text {out }}} Q(x, t) z(x)^{2} \mathrm{~d} \Gamma_{\text {out }} \geq 0$, since $Q$ is nonnegative by assumption, and $\int_{\Omega} c(x, t) p(x) q(x) \mathrm{d} x \geq-\frac{\|c\|_{L^{\infty}(\Omega \times(0, T))}}{2}\left(\|p\|_{L^{2}(\Omega)}^{2}+\|q\|_{L^{2}(\Omega)}^{2}\right)$.

Consequently,

$$
\begin{aligned}
\bar{A}(t,(p, q),(p, q)) \geq & \left(D_{\mathrm{S}}-\frac{1}{4 \epsilon_{1}}\|Q\|_{L^{\infty}(\bar{\Omega} \times(0, T))}\right)\|\nabla p\|_{L^{2}(\Omega)}^{2} \\
& -\left(\frac{\|c\|_{L^{\infty}(\Omega \times(0, T))}}{2}+\epsilon_{1}\|Q\|_{L^{\infty}(\bar{\Omega} \times(0, T))}\right)\|p\|_{L^{2}(\Omega)}^{2} \\
& +\left(D_{\mathrm{B}}-\frac{1}{4 \epsilon_{2}}\|Q\|_{L^{\infty}(\bar{\Omega} \times(0, T))}\right)\|\nabla q\|_{L^{2}(\Omega)}^{2} \\
& -\left(\frac{\|c\|_{L^{\infty}(\Omega \times(0, T))}}{2}+\epsilon_{2}\|Q\|_{L^{\infty}(\bar{\Omega} \times(0, T))}\right)\|q\|_{L^{2}(\Omega)}^{2} .
\end{aligned}
$$

We choose $\epsilon_{1}>0$ and $\epsilon_{2}>0$ such that

$$
\alpha_{1}=D_{\mathrm{S}}-\frac{1}{4 \epsilon_{1}}\|Q\|_{L^{\infty}(\bar{\Omega} \times(0, T))}>0, \text { and } \alpha_{2}=D_{\mathrm{B}}-\frac{1}{4 \epsilon_{2}}\|Q\|_{L^{\infty}(\bar{\Omega} \times(0, T))}>0 .
$$

Then, we choose $\lambda_{1}>0$ and $\lambda_{2}>0$ such that

$$
\begin{gathered}
\alpha_{3}=\lambda_{1}-\frac{\|c\|_{L^{\infty}(\Omega \times(0, T))}}{2}-\epsilon_{1}\|Q\|_{L^{\infty}(\bar{\Omega} \times(0, T))}>0 \text { and } \\
\alpha_{4}=\lambda_{2}-\frac{3\|c\|_{(\Omega \times(0, T))}}{2}-\epsilon_{2}\|Q\|_{L^{\infty}(\bar{\Omega} \times(0, T))}>0 .
\end{gathered}
$$

Therefore, choosing $\alpha=\min \left\{\alpha_{1}, \alpha_{2}, \alpha_{3}, \alpha_{4}\right\}$ and $\lambda=\lambda_{1}+\lambda_{2}$, one has that

$$
|\bar{A}(t,(p, q),(p, q))|+\lambda\|(p, q)\|_{\mathbf{L}^{2}(\Omega)}^{2} \geq \alpha\left(\|p\|_{H^{1}(\Omega)}^{2}+\|q\|_{H^{1}(\Omega)}^{2}\right)=\alpha\|\boldsymbol{\psi}\|_{\mathbf{H}^{1}(\Omega)}^{2} .
$$

Finally, in order to apply Theorem A.3 we need to prove that $f:(0, T) \longrightarrow\left(\mathbf{H}^{1}(\Omega)\right)^{\prime}$, with $f(t): \mathbf{H}^{1}(\Omega) \longrightarrow \mathbb{R}$ defined by

$$
\left(\begin{array}{c}
v \\
w
\end{array}\right) \rightarrow \int_{\Gamma_{\mathrm{in}}} Q(x, t) S_{\mathrm{e}}(t) v(x) \mathrm{d} \Gamma_{\mathrm{in}}
$$

is in $L^{2}\left(0, T, \mathbf{H}^{1}(\Omega)^{\prime}\right)$.

Firstly, we must see that $f(t)$ is linear and continuous a.e. $t \in(0, T)$. The linearity of $f(t)$ follows from the linearity of the integral. Because of this linearity, the continuity property is equivalent to the existence of $k(t)>0$ such that $|f(t)(\phi)| \leq k(t)\|\phi\|_{\mathbf{H}^{1}(\Omega)}, \forall \boldsymbol{\phi} \in \mathbf{H}^{1}(\Omega)$.

Now, given $\phi=\left(\begin{array}{l}u \\ v\end{array}\right)$, one has

$|f(t)(\phi)|=\left|\int_{\Gamma_{\text {in }}} Q(x, t) S_{\mathrm{e}}(t) v(x) \mathrm{d} \Gamma_{\text {in }}\right| \leq\|Q(\cdot, t)\|_{L^{\infty}(\bar{\Omega})}\left|S_{\mathrm{e}}(t)\right|\left|\Gamma_{\mathrm{in}}\right|^{\frac{1}{2}}\|v\|_{L^{2}\left(\Gamma_{\mathrm{in}}\right)}$

a.e. $t \in(0, T)$, where $\left|\Gamma_{\text {in }}\right|$ is the Lebesgue measure of $\Gamma_{\text {in }}$. Using the Trace Theorem A.9, we conclude that there exists a constant $C_{\mathrm{T}}>0$ such that:

$$
|f(t)(\phi)| \leq \underbrace{C_{\mathrm{T}}\left|\Gamma_{\mathrm{in}}\right|^{\frac{1}{2}}\|Q(\cdot, t)\|_{L^{\infty}(\bar{\Omega})}\left|S_{\mathrm{e}}(t)\right|}_{(:=k(t))}\|v\|_{H^{1}(\Omega)} \leq k(t)\|\phi\|_{\mathbf{H}^{1}(\Omega)} .
$$


Secondly, we must see that $\int_{0}^{T}\|f(t)\|_{\left(\mathbf{H}^{1}(\Omega)\right)^{\prime}}^{2} \mathrm{~d} t<\infty$. We use that

$$
\|G\|_{\left(\mathbf{H}^{1}(\Omega)\right)^{\prime}}=\sup _{\substack{\boldsymbol{\phi} \in \mathbf{H}^{1}(\Omega) \\\|\boldsymbol{\phi}\| \leq 1}}|<G, \boldsymbol{\phi}\rangle \mid
$$

and thus, by the hypothesis on $Q$ and $S_{\mathrm{e}}$, we have that

$$
\begin{aligned}
\int_{0}^{T}\|f(t)\|_{\left(\mathbf{H}^{1}(\Omega)\right)^{\prime}}^{2} \mathrm{~d} t & \leq \int_{0}^{T}\left|\Gamma_{\mathrm{in}}\right| C_{\mathrm{T}}^{2}\left|S_{\mathrm{e}}(t)\right|^{2}\|Q(\cdot, t)\|_{L^{\infty}(\bar{\Omega})} \mathrm{d} t \\
& \leq\left|\Gamma_{\mathrm{in}}\right| C_{\mathrm{T}}^{2}\|Q\|_{L^{\infty}(\bar{\Omega} \times(0, T))}^{2}\left\|S_{\mathrm{e}}\right\|_{L^{2}(0, T)}^{2}<\infty .
\end{aligned}
$$

Since we have proved that all the assumptions of Theorem A.3 are satisfied, the proof of Theorem 3.4 is finished.

Before proving Theorem 3.1, we prove the following result:

Proposition 3.5. If $(S, B)$ is the weak solution of System (3), then

$$
\|S\|_{W\left(0, T, H^{1}(\Omega),\left(H^{1}(\Omega)\right)^{\prime}\right)} \leq C \text { and }\|B\|_{W\left(0, T, H^{1}(\Omega),\left(H^{1}(\Omega)\right)^{\prime}\right)} \leq C,
$$

where $C$ depends on $D_{\mathrm{S}}, D_{\mathrm{B}},\left\|S_{\text {init }}\right\|_{L^{2}(\Omega)},\left\|B_{\text {init }}\right\|_{L^{2}(\Omega)},\|Q\|_{L^{\infty}(\bar{\Omega} \times(0, T))},\left\|S_{\mathrm{e}}\right\|_{L^{2}(0, T)},\left|\Gamma_{\mathrm{in}}\right|, T$, $\|c\|_{L^{\infty}(\Omega \times(0, T))}$ and $C_{\mathrm{T}}$ (i.e., the constant coming from the Trace Theorem A.9).

Proof of Proposition 3.5. From the first equation in System (3), it follows that

$$
\begin{aligned}
& \left\|\frac{\mathrm{d} S}{\mathrm{~d} t}\right\|_{L^{2}\left(0, T,\left(H^{1}(\Omega)\right)^{\prime}\right)}=\sup _{\phi \in L^{2}\left(0, T, H^{1}(\Omega)\right),}^{\|\phi\| \leq 1},\left|<\frac{\mathrm{d} S}{\mathrm{~d} t}, \phi>\right| \\
& =\sup _{\substack{\phi L^{2}\left(0, T, H^{1}(\Omega)\right),\|\phi\| \leq 1}}\left|<\operatorname{div}\left(D_{\mathrm{S}} \nabla S-\mathbf{Q} S\right)-c B, \phi>\right| .
\end{aligned}
$$

Given $\phi \in L^{2}\left(0, T, H^{1}(\Omega)\right)$, one has

$$
\begin{aligned}
& \left|<\operatorname{div}\left(D_{\mathrm{S}} \nabla S-\mathbf{Q} S\right)-c B, \phi>\right| \\
& =\mid \int_{0}^{T} \int_{\delta \Omega} \mathbf{n} \cdot\left(D_{\mathrm{S}} \nabla S(x, \tau)-\mathbf{Q}(x, \tau) S(x, \tau)\right) \phi(x, \tau) \mathrm{d} x \mathrm{~d} \tau \\
& \quad-D_{\mathrm{S}} \int_{0}^{T} \int_{\Omega} \nabla S(x, \tau) \nabla \phi(x, \tau) \mathrm{d} x \mathrm{~d} \tau+\int_{0}^{T} \int_{\Omega} \mathbf{Q} S(x, \tau) \nabla \phi(x, \tau) \mathrm{d} x \mathrm{~d} \tau \\
& \quad-\int_{0}^{T} \int_{\Omega} c(x, \tau) B(x, \tau) \phi(x, \tau) \mathrm{d} x \mathrm{~d} \tau \mid \\
& \leq\|Q\|_{L^{\infty}(\bar{\Omega} \times(0, T))}\left\|S_{\mathrm{e}}\right\|_{L^{2}(0, T)}\left|\Gamma_{\text {in }}\right|^{\frac{1}{2}}\|\phi\|_{L^{2}\left(0, t, L^{2}\left(\Gamma_{\text {in }}\right)\right)} \\
& \quad+\|Q\|_{L^{\infty}(\bar{\Omega} \times(0, T))}\|S\|_{L^{2}\left(0, T, L^{2}\left(\Gamma_{\text {out }}\right)\right)}\|\phi\|_{L^{2}\left(0, T, L^{2}\left(\Gamma_{\text {out }}\right)\right)} \\
& \quad+D_{\mathrm{S}}\|\nabla \phi\|_{L^{2}\left(0, T, L^{2}(\Omega)\right)}\|\nabla S\|_{L^{2}\left(0, T, L^{2}(\Omega)\right)} \\
& \quad+\|Q\|_{L^{\infty}(\bar{\Omega} \times(0, T))}\|S\|_{L^{2}\left(0, T, L^{2}(\Omega)\right)}\|\nabla \phi\|_{L^{2}\left(0, T, L^{2}(\Omega)\right)} \\
& \quad+\|c\|_{L^{\infty}(\Omega \times(0, T))}\|B\|_{L^{2}\left(0, T, L^{2}(\Omega)\right)}\|\phi\|_{L^{2}\left(0, T, L^{2}(\Omega)\right)} .
\end{aligned}
$$

Consequently, if $C_{\mathrm{T}}$ is the constant coming from the Trace Theorem A.9, one has that

$$
\begin{aligned}
\left\|\frac{\mathrm{d} S}{\mathrm{~d} t}\right\|_{L^{2}\left(0, T,\left(H^{1}(\Omega)\right)^{\prime}\right)} \leq & C_{\mathrm{T}}\|Q\|_{L^{\infty}(\bar{\Omega} \times(0, T))}\left\|S_{\mathrm{e}}\right\|_{L^{2}(0, T)}\left|\Gamma_{\mathrm{in}}\right|^{\frac{1}{2}} \\
& +\|c\|_{L^{\infty}(\Omega \times(0, T))}\|B\|_{L^{2}\left(0, T, L^{2}(\Omega)\right)} \\
& \left(D_{\mathrm{S}}+\left(1+C_{\mathrm{T}}^{2}\right)\|Q\|_{L^{\infty}(\bar{\Omega} \times(0, T))}\right)\|S\|_{L^{2}\left(0, T, H^{1}(\Omega)\right)} .
\end{aligned}
$$


Similarly, from the second equation in System (3), it follows that $\left\|\frac{\mathrm{d} B}{\mathrm{~d} t}\right\|_{L^{2}\left(0, T,\left(H^{1}(\Omega)\right)^{\prime}\right)}=\sup _{\substack{\phi \in L^{2}\left(0, T, H^{1}(\Omega)\right) \\\|\phi\| \leq 1}}\left|<\operatorname{div}\left(D_{\mathrm{B}} \nabla B-\mathbf{Q} B\right)+c B, \phi>\right|$. Given $\phi \in L^{2}\left(0, T, H^{1}(\Omega)\right)$, one has

$$
\begin{aligned}
\mid<\operatorname{div}\left(D_{\mathrm{B}} \nabla B-\mathbf{Q}\right) B & +c B, \phi>\mid \\
& \leq\|Q\|_{L^{\infty}(0, T)}\|B\|_{L^{2}\left(0, T, L^{2}\left(\Gamma_{\text {out }}\right)\right)}\|\phi\|_{L^{2}\left(0, T, L^{2}\left(\Gamma_{\text {out }}\right)\right)} \\
& +D_{\mathrm{B}}\|\nabla \phi\|_{L^{2}\left(0, T, L^{2}(\Omega)\right)}\|\nabla B\|_{L^{2}\left(0, T, L^{2}(\Omega)\right)} \\
& +\|Q\|_{L^{\infty}(\bar{\Omega} \times(0, T))}\|B\|_{L^{2}\left(0, T, L^{2}(\Omega)\right)}\|\nabla \phi\|_{L^{2}\left(0, T, L^{2}(\Omega)\right)} \\
& +\|c\|_{L^{\infty}(\Omega \times(0, T))}\|B\|_{L^{2}\left(0, T, L^{2}(\Omega)\right)}\|\phi\|_{L^{2}\left(0, T, L^{2}(\Omega)\right)} .
\end{aligned}
$$

Again using the Trace Theorem A.9, one has that

$$
\begin{aligned}
& \left\|\frac{\mathrm{d} B}{\mathrm{~d} t}\right\|_{L^{2}\left(0, T,\left(H^{1}(\Omega)\right)^{\prime}\right)} \\
& \quad \leq\left(D_{\mathrm{B}}+\left(1+C_{\mathrm{T}}^{2}\right)\|Q\|_{L^{\infty}(\bar{\Omega} \times(0, T))}+\|c\|_{L^{\infty}(\Omega \times(0, T))}\right)\|B\|_{L^{2}\left(0, T, H^{1}(\Omega)\right)}
\end{aligned}
$$

Now, in order to obtain estimates for $\|S\|_{L^{2}\left(0, T, H^{1}(\Omega)\right)}$ and $\|B\|_{L^{2}\left(0, T, H^{1}(\Omega)\right)}$, we consider $\lambda \geq 0$ and the variables $\bar{S}=e^{-\lambda t} S$ and $\bar{B}=e^{-\lambda t} B$ that fulfill

$$
\begin{aligned}
& \bar{S}_{t}+\lambda \bar{S}-\operatorname{div}\left(D_{\mathrm{S}} \nabla \bar{S}-\mathbf{Q} \bar{S}\right)+c \bar{B}=0 \\
& \bar{B}_{t}+\lambda \bar{B}-\operatorname{div}\left(D_{\mathrm{B}} \nabla \bar{S}-\mathbf{Q} \bar{B}\right)+c \bar{B}=0 .
\end{aligned}
$$

Multiplying the first equation in (7) by $\bar{S}$ and integrating, one obtains

$$
\begin{aligned}
& \frac{1}{2}\|\bar{S}(T)\|_{L^{2}(\Omega)}^{2}+\lambda \int_{0}^{T}\|\bar{S}(\tau)\|_{L^{2}(\Omega)}^{2} \mathrm{~d} \tau+\int_{0}^{T} \int_{\Gamma_{\text {out }}} Q(x, \tau) \bar{S}^{2}(x, \tau) \mathrm{d} x \mathrm{~d} \tau \\
&+D_{\mathrm{S}} \int_{0}^{T}\|\nabla \bar{S}(\tau)\|_{L^{2}(\Omega)}^{2} \mathrm{~d} \tau= \frac{1}{2}\left\|S_{\text {init }}\right\|_{L^{2}(\Omega)}^{2} \\
&+\int_{0}^{T} e^{-\lambda \tau} \int_{\Gamma_{\text {in }}} Q(x, \tau) S_{\mathrm{e}}(\tau) \bar{S}(x, \tau) \mathrm{d} x \mathrm{~d} \tau \\
&+\int_{0}^{T} \int_{\Omega} \mathbf{Q}(x, \tau) \bar{S}(x, \tau) \nabla \bar{S}(x, \tau) \mathrm{d} x \mathrm{~d} \tau \\
&-\int_{0}^{T} \int_{\Omega} c(x, \tau) \bar{S}(x, \tau) \bar{B}(x, \tau) \mathrm{d} x \mathrm{~d} \tau .
\end{aligned}
$$

Applying Young's Inequality (36) with $\epsilon_{1}>0$ and the Trace Theorem A.9,

$\int_{0}^{T} \int_{\Gamma_{\text {in }}} e^{-\lambda \tau} Q(x, \tau) S_{\mathrm{e}}(\tau) \bar{S}(\tau, x) \mathrm{d} x \mathrm{~d} \tau$

$\leq \int_{0}^{T} e^{-\lambda \tau}\left|Q(x, \tau)\left\|\left.S_{\mathrm{e}}(\tau)\left|\|\bar{S}(\tau)\|_{L^{2}\left(\Gamma_{\text {in }}\right)}\right| \Gamma_{\text {in }}\right|^{\frac{1}{2}} \mathrm{~d} \tau\right.\right.$

$\leq\left|\Gamma_{\text {in }}\right|^{\frac{1}{2}}\|Q\|_{L^{\infty}(\bar{\Omega} \times(0, T))}\left(\epsilon_{1}\left\|S_{\mathrm{e}}\right\|_{L^{2}(0, T)}^{2}+\frac{C_{\mathrm{T}}^{2}}{4 \epsilon_{1}}\|\bar{S}\|_{L^{2}\left(0, T, L^{2}(\Omega)\right)}^{2}+\frac{C_{\mathrm{T}}^{2}}{4 \epsilon_{1}}\|\nabla \bar{S}\|_{L^{2}\left(0, T, L^{2}(\Omega)\right)}^{2}\right)$.

Applying Young's Inequality (36) again, with $\epsilon_{2}>0$, 
$\int_{0}^{T} \int_{\Omega} \mathbf{Q}(x, \tau) \bar{S}(x, \tau) \nabla \bar{S}(x, \tau) \mathrm{d} x \mathrm{~d} \tau$

$$
\begin{aligned}
& \leq\|Q\|_{L^{\infty}(\bar{\Omega} \times(0, T))}\|\bar{S}\|_{L^{2}\left(0, T, L^{2}(\Omega)\right.}\|\nabla \bar{S}\|_{L^{2}\left(0, T, L^{2}(\Omega)\right.} \\
& \leq \epsilon_{2}\|Q\|_{L^{\infty}(\bar{\Omega} \times(0, T))}\|\bar{S}\|_{L^{2}\left(0, T, L^{2}(\Omega)\right.}^{2}+\frac{\|Q\|_{L^{\infty}(\bar{\Omega} \times(0, T))}}{4 \epsilon_{2}}\|\nabla \bar{S}\|_{L^{2}\left(0, T, L^{2}(\Omega)\right.}^{2} .
\end{aligned}
$$

Moreover, applying Young's Inequality (36) with $\epsilon=\frac{1}{2}$,

$\int_{0}^{T} \int_{\Omega} c(x, \tau) \bar{S}(x, \tau) \bar{B}(x, \tau) \mathrm{d} x \mathrm{~d} \tau$

$$
\leq\|c\|_{L^{\infty}(\Omega \times(0, T))}\left(\frac{\|\bar{S}\|_{L^{2}\left(0, T, L^{2}(\Omega)\right)}^{2}}{2}+\frac{\|\bar{B}\|_{L^{2}\left(0, T, L^{2}(\Omega)\right)}^{2}}{2}\right) .
$$

Thus, considering (8), one has

$$
\begin{aligned}
& \frac{1}{2}\|\bar{S}(T)\|_{L^{2}(\Omega)}^{2}+\left(D_{\mathrm{S}}-\|Q\|_{L^{\infty}(\bar{\Omega} \times(0, T))}\left(\frac{1}{4 \epsilon_{2}}+\frac{\left|\Gamma_{\mathrm{in}}\right|^{\frac{1}{2}} C_{\mathrm{T}}^{2}}{4 \epsilon_{1}}\right)\right)\|\nabla \bar{S}(\tau)\|_{L^{2}\left(0, T, L^{2}(\Omega)\right)}^{2} \\
& +\left(\lambda-\|Q\|_{L^{\infty}(\bar{\Omega} \times(0, T))}\left(\epsilon_{2}+\frac{\left|\Gamma_{\mathrm{in}}\right|^{\frac{1}{2}} C_{\mathrm{T}}^{2}}{4 \epsilon_{1}}\right)-\frac{\|c\|_{L^{\infty}(\Omega \times(0, T))}}{2}\right)\|\bar{S}\|_{L^{2}\left(0, T, L^{2}(\Omega)\right)}^{2} \\
& \leq \frac{1}{2}\left\|S_{\text {init }}\right\|_{L^{2}(\Omega)}^{2}+\epsilon_{1}\|Q\|_{L^{\infty}(\bar{\Omega} \times(0, T))}\left\|S_{\mathrm{e}}\right\|_{L^{2}(0, T)}^{2}\left|\Gamma_{\mathrm{in}}\right|^{\frac{1}{2}}+\frac{\|c\|_{L^{\infty}(\Omega \times(0, T))}\|\bar{B}\|_{L^{2}\left(0, T, L^{2}(\Omega)\right)}^{2}}{2}
\end{aligned}
$$

Analogously, if we multiply the second equation in (7) by $\bar{B}$ and we integrate, we obtain

$$
\begin{gathered}
\frac{1}{2}\|\bar{B}(T)\|_{L^{2}(\Omega)}^{2}+\left(\lambda-\epsilon_{3}\|Q\|_{L^{\infty}(\bar{\Omega} \times(0, T))}-\|c\|_{L^{\infty}(\Omega \times(0, T))}\right)\|\bar{B}\|_{L^{2}\left(0, T, L^{2}(\Omega)\right)}^{2} \\
+\left(D_{\mathrm{B}}-\frac{\|Q\|_{L^{\infty}(\bar{\Omega} \times(0, T))}}{4 \epsilon_{3}}\right)\|\nabla \bar{B}(\tau)\|_{L^{2}\left(0, T, L^{2}(\Omega)\right)}^{2} \leq \frac{1}{2}\left\|B_{\text {init }}\right\|_{L^{2}(\Omega)}^{2} .
\end{gathered}
$$

Choosing $\epsilon_{1}$ and $\epsilon_{2}$ such that $D_{\mathrm{S}} \geq\|Q\|_{L^{\infty}(\bar{\Omega} \times(0, T))}\left(\frac{1}{4 \epsilon_{2}}+\frac{\left|\Gamma_{\mathrm{in}}\right|^{\frac{1}{2}} C_{\mathrm{T}}^{2}}{4 \epsilon_{1}}\right), \epsilon_{3} \geq \frac{\|Q\|_{L^{\infty}(\bar{\Omega} \times(0, T))}}{4 D_{\mathrm{B}}}$ and $\lambda>\|Q\|_{L^{\infty}(\bar{\Omega} \times(0, T))} \max \left(\epsilon_{3}, \epsilon_{2}+\frac{C_{\mathrm{T}}^{2}\left|\Gamma_{\text {in }}\right|^{\frac{1}{2}}}{4 \epsilon_{1}}\right)+\|c\|_{L^{\infty}(\Omega \times(0, T))}$, it follows that

$$
\begin{aligned}
\|\bar{B}\|_{L^{2}\left(0, T, H^{1}(\Omega)\right)}^{2} \leq & \alpha_{1}\left\|B_{\text {init }}\right\|_{L^{2}(\Omega)}^{2}, \\
\|\bar{S}\|_{L^{2}\left(0, T, H^{1}(\Omega)\right)}^{2} \leq & \alpha_{2}\left(\frac{1}{2}\left\|S_{\text {init }}\right\|_{L^{2}(\Omega)}^{2}+\epsilon_{1}\|Q\|_{L^{\infty}(\bar{\Omega} \times(0, T))}\left\|S_{\mathrm{e}}\right\|_{L^{2}(0, T)}^{2}\left|\Gamma_{\text {in }}\right|^{\frac{1}{2}}\right) \\
& +\alpha_{1} \alpha_{2} \frac{\|c\|_{L^{\infty}(\Omega \times(0, T))}}{2}\left\|B_{\text {init }}\right\|_{L^{2}(\Omega)}^{2},
\end{aligned}
$$

where $\alpha_{1}, \alpha_{2}>0$ depend on $\left|\Gamma_{\text {in }}\right|,\|c\|_{L^{\infty}(\Omega \times(0, T))},\|Q\|_{L^{\infty}(0, T)}, C_{\mathrm{T}}, D_{\mathrm{S}}$ and $D_{\mathrm{B}}$.

Furthermore, it is straight forward to see that

$$
\begin{gathered}
\|B\|_{L^{2}\left(0, T, L^{2}(\Omega)\right)}^{2} \leq e^{\lambda T}\|\bar{B}\|_{L^{2}\left(0, T, L^{2}(\Omega)\right)}^{2}, \\
\|S\|_{L^{2}\left(0, T, H^{1}(\Omega)\right)}^{2} \leq e^{\lambda T}\|\bar{S}\|_{L^{2}\left(0, T, H^{1}(\Omega)\right)}^{2} .
\end{gathered}
$$

From (5), (11) and (12), it follows that

$$
\|S\|_{W\left(0, T, H^{1}(\Omega),\left(H^{1}(\Omega)\right)^{\prime}\right)},\|B\|_{W\left(0, T, H^{1}(\Omega),\left(H^{1}(\Omega)\right)^{\prime}\right)} \leq C,
$$

where $C=C\left(T,\left\|S_{\text {init }}\right\|_{L^{2}(\Omega)},\left\|B_{\text {init }}\right\|_{L^{2}(\Omega)}, D_{\mathrm{S}}, D_{\mathrm{B}},\|Q\|_{L^{\infty}(\bar{\Omega} \times(0, T))},\left\|S_{\mathrm{e}}\right\|_{L^{2}(0, T)}\right.$, $\left.\|c\|_{L^{\infty}(\Omega \times(0, T))},\left|\Gamma_{\mathrm{in}}\right|, C_{\mathrm{T}}\right)$. 
Proof of Theorem 3.1. In order to prove the existence of solution, we apply Schauder Fixed Point Theorem A.4. We have to choose a Banach space $X$ and a compact and convex subset $K \subset X$.

We consider the Banach Space $W\left(0, T, H^{1}(\Omega),\left(H^{1}(\Omega)\right)^{\prime}\right)$, which is compactly embedded in $L^{2}\left(0, T, L^{2}(\Omega)\right)$ (see Remark $\left.\mathbf{A . 8}\right)$.

If $Z \in W\left(0, T, H^{1}(\Omega),\left(H^{1}(\Omega)\right)^{\prime}\right)$ and we solve the linear System (3) with $c(x, t)=\mu(Z(x, t))$, Theorem 3.4 proves that there exists a unique weak solution $\left(S_{\mathrm{Z}}, B_{\mathrm{Z}}\right)$ with $S_{\mathrm{Z}}, B_{\mathrm{Z}} \in W\left(0, T, H^{1}(\Omega),\left(H^{1}(\Omega)\right)^{\prime}\right)$. Furthermore, Proposition 3.5 shows that

$$
\left\|B_{Z}\right\|_{W\left(0, T, H^{1}(\Omega),\left(H^{1}(\Omega)\right)^{\prime}\right)} \leq C \text { and }\left\|S_{Z}\right\|_{W\left(0, T, H^{1}(\Omega),\left(H^{1}(0, T)\right)^{\prime}\right)} \leq C,
$$

where $C$ depends (among others) on the norm of $\mu(Z(x, t))$. Since $\mu(\cdot) \in L^{\infty}(\mathbb{R})$ it follows that for all $Z \in W\left(0, T, H^{1}(\Omega),\left(H^{1}(\Omega)\right)^{\prime}\right)$, we have

$$
\left\|B_{Z}\right\|_{W\left(0, T, H^{1}(\Omega),\left(H^{1}(\Omega)\right)^{\prime}\right)} \leq \bar{C} \text { and }\left\|S_{Z}\right\|_{W\left(0, T, H^{1}(\Omega),\left(H^{1}(\Omega)\right)^{\prime}\right)} \leq \bar{C},
$$

where $\bar{C}$ is a constant depending (among others) on $\|\mu\|_{L^{\infty}(\mathbb{R})}$.

If we define the set

$$
K:=\left\{z \in W\left(0, T, H^{1}(\Omega),\left(H^{1}(\Omega)\right)^{\prime}\right):\|z\|_{W\left(0, T, H^{1}(\Omega),\left(H^{1}(\Omega)\right)^{\prime}\right)} \leq \bar{C}\right\},
$$

from Remark A.8 and Definition A.5, $K$ is a compact set of the Banach Space $X:=L^{2}\left(0, T, L^{2}(\Omega)\right)$.

Let us define the application $A: K \rightarrow K$ by $A(Z)=S_{\mathrm{Z}}$. We prove Theorem 3.1 by showing that $A$ has a fixed point. In order to apply Schauder Fixed Point Theorem, it is enough to prove that $A$ is continuous.

In this direction, if $\left\{Z_{\mathrm{n}}\right\}_{n} \subset K, Z \in K$ are such that $\left\|Z_{\mathrm{n}}-Z\right\|_{X} \stackrel{n \rightarrow \infty}{\longrightarrow} 0$, we must prove that

$$
\left\|A\left(Z_{\mathrm{n}}\right)-A(Z)\right\|_{X}=\left\|S_{Z_{\mathrm{n}}}-S_{\mathrm{Z}}\right\|_{L^{2}\left(0, T, L^{2}(\Omega)\right)} \stackrel{n \rightarrow \infty}{\longrightarrow} 0 .
$$

Let $\left(S_{\mathrm{Z}_{\mathrm{n}}}, B_{\mathrm{Z}_{\mathrm{n}}}\right)$ and $\left(S_{\mathrm{Z}}, B_{\mathrm{Z}}\right)$ be the weak solutions of linear system (3) when $c(x, t)=$ $\mu\left(Z_{\mathrm{n}}(x, t)\right)$ and $c(x, t)=\mu(Z(x, t))$, respectively. We denote $V_{n}=S_{\mathrm{Z}_{\mathrm{n}}}-S_{\mathrm{Z}}$ and $W_{n}=$ $B_{Z_{\mathrm{n}}}-B_{\mathrm{Z}}$. Then $\left(V_{n}, W_{n}\right)$ is a weak solution of:

$$
\begin{cases}\left(V_{n}\right)_{t}-\operatorname{div}\left(D_{\mathrm{S}} \nabla V_{n}-\mathbf{Q} V_{n}\right)+\mu(Z) B_{\mathrm{Z}}-\mu\left(Z_{\mathrm{n}}\right) B_{\mathrm{Z}_{\mathrm{n}}}=0 & \text { in } \Omega \times(0, T), \\ \left(W_{n}\right)_{t}-\operatorname{div}\left(D_{\mathrm{B}} \nabla W_{n}-\mathbf{Q} W_{n}\right)-\mu(Z) B_{\mathrm{Z}}+\mu\left(Z_{\mathrm{n}}\right) B_{\mathrm{Z}_{\mathrm{n}}}=0 & \text { in } \Omega \times(0, T),\end{cases}
$$

and the initial and boundary conditions

$$
\left\{\begin{array}{lr}
V_{n}(x, 0)=0 & \text { in } \Omega, \\
W_{n}(x, 0)=0 & \text { in } \Omega, \\
\mathbf{n} \cdot\left(D_{\mathrm{S}} \nabla V_{n}-\mathbf{Q} V_{n}\right)=0 & \text { in } \Gamma_{\text {in }} \cup \Gamma_{\text {wall }} \times(0, T), \\
\mathbf{n} \cdot\left(D_{\mathrm{B}} \nabla W_{n}-\mathbf{Q} W_{n}\right)=0 & \text { in } \Gamma_{\text {in }} \cup \Gamma_{\text {wall }} \times(0, T), \\
\mathbf{n} \cdot\left(D_{\mathrm{S}} \nabla V_{n}\right)=0 & \text { in } \Gamma_{\text {out }} \times(0, T), \\
\mathbf{n} \cdot\left(D_{\mathrm{B}} \nabla W_{n}\right)=0 & \text { in } \Gamma_{\text {out }} \times(0, T) .
\end{array}\right.
$$


Given $\lambda>0$, then $\bar{V}_{n}=e^{-\lambda t} V_{n}$ and $\bar{W}_{n}=e^{-\lambda t} W_{n}$ fulfill:

$$
\begin{aligned}
& \left(\bar{V}_{n}\right)_{\mathrm{t}}+\lambda \bar{V}_{n}-\operatorname{div}\left(D_{\mathrm{S}} \nabla \bar{V}_{n}-\mathbf{Q} \bar{V}_{n}\right)+e^{-\lambda t}\left(\mu(Z) B_{\mathrm{Z}}-\mu\left(Z_{\mathrm{n}}\right) B_{\mathrm{Z}_{\mathrm{n}}}\right)=0, \\
& \left(\bar{W}_{n}\right)_{\mathrm{t}}+\lambda \bar{W}_{n}-\operatorname{div}\left(D_{\mathrm{B}} \nabla \bar{W}_{n}-\mathbf{Q} \bar{W}_{n}\right)-e^{-\lambda t}\left(\mu(Z) B_{\mathrm{Z}}-\mu\left(Z_{\mathrm{n}}\right) B_{\mathrm{Z}_{\mathrm{n}}}\right)=0 .
\end{aligned}
$$

Multiplying the first equation of (14) by $\bar{V}_{n}$ and integrating, one obtains:

$\frac{1}{2}\left\|\bar{V}_{n}(T)\right\|_{L^{2}(\Omega)}^{2}+\lambda \int_{0}^{T}\left\|\bar{V}_{n}(\tau)\right\|_{L^{2}(\Omega)}^{2} \mathrm{~d} \tau+\int_{0}^{T} \int_{\Gamma_{\text {out }}} Q(x, \operatorname{tau}) \bar{V}_{n}^{2}(x, \tau) \mathrm{d} x \mathrm{~d} \tau$ $\frac{1}{2}\left\|\bar{V}_{n}(T)\right\|_{L^{2}(\Omega)}^{2}+\lambda \int_{0}^{T}\left\|\bar{V}_{n}(\tau)\right\|_{L^{2}(\Omega)}^{2} \mathrm{~d} \tau+\int_{0}^{T} \int_{\Gamma_{\text {out }}} Q(x, \tau) \bar{V}_{n}^{2}(x, \tau) \mathrm{d} x \mathrm{~d} \tau$

$+D_{\mathrm{S}} \int_{0}^{T}\left\|\nabla \bar{V}_{n}(\tau)\right\|_{L^{2}(\Omega)}^{2} \mathrm{~d} \tau=\int_{0}^{T} \int_{\Omega} \mathbf{Q}(x, \tau) \bar{V}_{n}(x, \tau) \nabla \bar{V}_{n}(x, \tau) \mathrm{d} x \mathrm{~d} \tau$

$+\int_{0}^{T} e^{-\lambda \tau} \int_{\Omega}\left(\mu\left(Z_{\mathrm{n}}(x, \tau)\right) B_{\mathrm{Z}_{\mathrm{n}}}(x, \tau)-\mu(Z(x, \tau)) B_{\mathrm{Z}}(x, \tau)\right) \bar{V}_{n}(x, \tau) \mathrm{d} x \mathrm{~d} \tau$.

Similarly, if we multiply the second equation in (14) by $\bar{W}_{n}$, we have $\frac{1}{2}\left\|\bar{W}_{n}(T)\right\|_{L^{2}(\Omega)}^{2}+\lambda \int_{0}^{T}\left\|\bar{W}_{n}(\tau)\right\|_{L^{2}(\Omega)}^{2} \mathrm{~d} \tau+\int_{0}^{T} \int_{\Gamma_{\text {out }}} Q(x, \tau) \bar{W}_{n}^{2}(x, \tau) \mathrm{d} x \mathrm{~d} \tau$ $+D_{\mathrm{B}} \int_{0}^{T}\left\|\nabla \bar{W}_{n}(\tau)\right\|_{L^{2}(\Omega)}^{2} \mathrm{~d} \tau=\int_{0}^{T} \int_{\Omega} \mathbf{Q}(x, \tau) \bar{W}_{n}(x, \tau) \nabla \bar{W}_{n}(x, \tau) \mathrm{d} x \mathrm{~d} \tau$ $+\int_{0}^{T} e^{-\lambda \tau} \int_{\Omega}\left(\mu(Z(x, \tau)) B_{\mathrm{Z}}(x, \tau)-\mu\left(Z_{\mathrm{n}}(x, \tau)\right) B_{\mathrm{Z}_{\mathrm{n}}}(x, \tau)\right) \bar{W}_{n}(x, \tau) \mathrm{d} x \mathrm{~d} \tau$.

Summing equations (15) and (16) it follows:

$$
\begin{aligned}
& \frac{1}{2}\left(\left\|\bar{V}_{n}(T)\right\|_{L^{2}(\Omega)}^{2}+\left\|\bar{W}_{n}(T)\right\|_{L^{2}(\Omega)}^{2}\right)+\lambda \int_{0}^{T}\left(\left\|\bar{V}_{n}(\tau)\right\|_{L^{2}(\Omega)}^{2}+\left\|\bar{W}_{n}(\tau)\right\|_{L^{2}(\Omega)}^{2}\right) \mathrm{d} \tau \\
& +\int_{0}^{T}\|Q(\cdot, \tau)\|_{L^{\infty}(\bar{\Omega})}\left(\left\|\bar{V}_{n}(\tau)\right\|_{L^{2}\left(\Gamma_{\text {out }}\right)}^{2}+\left\|\bar{W}_{n}(\tau)\right\|_{L^{2}\left(\Gamma_{\text {out }}\right)}^{2}\right) \mathrm{d} \tau \\
& +\int_{0}^{T}\left(D_{\mathrm{S}}\left\|\nabla \bar{V}_{n}(\tau)\right\|_{L^{2}(\Omega)}^{2}+D_{\mathrm{B}}\left\|\nabla \bar{W}_{n}(\tau)\right\|_{L^{2}(\Omega)}^{2}\right) \mathrm{d} \tau \\
& =\int_{0}^{T} \int_{\Omega} \mathbf{Q}(x, \tau)\left(\bar{V}_{n}(x, \tau) \nabla \bar{V}_{n}(x, \tau)+\bar{W}_{n}(x, \tau) \nabla \bar{W}_{n}(x, \tau)\right) \mathrm{d} x \mathrm{~d} \tau \\
& +\int_{0}^{T} e^{-\lambda \tau} \int_{\Omega}\left(\mu(Z(x, \tau)) B_{\mathrm{Z}}(x, \tau)-\mu\left(Z_{\mathrm{n}}(x, \tau)\right) B_{\mathrm{Z}_{\mathrm{n}}}(x, \tau)\right)\left(\bar{W}_{n}(x, \tau)-\bar{V}_{n}(x, \tau)\right) \mathrm{d} x \mathrm{~d} \tau
\end{aligned}
$$


For the last term in (17) we have that

$$
\begin{aligned}
& \int_{0}^{T} e^{-\lambda \tau} \int_{\Omega}\left(\mu(Z(x, \tau)) B_{\mathrm{Z}}(x, \tau)-\mu\left(Z_{\mathrm{n}}(x, \tau)\right) B_{\mathrm{Z}_{\mathrm{n}}}(x, \tau)\right)\left(\bar{W}_{n}(x, \tau)-\bar{V}_{n}(x, \tau)\right) \mathrm{d} x \mathrm{~d} \tau \\
& =\int_{0}^{T} e^{-\lambda \tau} \int_{\Omega} \mu(Z(x, \tau))\left(B_{\mathrm{Z}}(x, \tau)-B_{\mathrm{Z}_{\mathrm{n}}}(x, \tau)\right)\left(\bar{W}_{n}(x, \tau)-\bar{V}_{n}(x, \tau)\right) \mathrm{d} x \mathrm{~d} \tau \\
& +\int_{0}^{T} e^{-\lambda \tau} \int_{\Omega} B_{\mathrm{Z}_{\mathrm{n}}}(x, \tau)\left(\mu(Z(x, \tau))-\mu\left(Z_{\mathrm{n}}(x, \tau)\right)\right)\left(\bar{W}_{n}(x, \tau)-\bar{V}_{n}(x, \tau)\right) \mathrm{d} x \mathrm{~d} \tau \\
& \leq \frac{3}{2}\|\mu\|_{L^{\infty}(\mathbb{R})} \int_{0}^{T}\left\|\bar{W}_{n}(\tau)\right\|_{L^{2}(\Omega)}^{2} \mathrm{~d} \tau+\frac{1}{2}\|\mu\|_{L^{\infty}(\mathbb{R})} \int_{0}^{T}\left\|\bar{V}_{n}(\tau)\right\|_{L^{2}(\Omega)}^{2} \mathrm{~d} \tau \\
& \quad+\int_{0}^{T}\left|\mu(Z(x, \tau))-\mu\left(Z_{\mathrm{n}}(x, \tau)\right)\left\|B_{\mathrm{Z}_{\mathrm{n}}}(x, \tau)\right\| \bar{W}_{n}(x, \tau)-\bar{V}_{n}(x, \tau)\right| \mathrm{d} x \mathrm{~d} \tau .
\end{aligned}
$$

Moreover, by applying Young's Inequality (36) with $\epsilon_{1}$, which will be chosen below, it follows

$\int_{0}^{T} \int_{\Omega} \mathbf{Q}(x, \tau) \bar{V}_{n}(x, \tau) \nabla \bar{V}_{n}(x, \tau) \mathrm{d} x \mathrm{~d} \tau$

$$
\leq\|Q\|_{L^{\infty}(\bar{\Omega} \times(0, T))} \int_{0}^{T}\left(\epsilon_{1}\left\|\bar{V}_{n}(\tau)\right\|_{L^{2}(\Omega)}^{2}+\frac{1}{4 \epsilon_{1}}\left\|\nabla \bar{V}_{n}(\tau)\right\|_{L^{2}(\Omega)}^{2}\right) \mathrm{d} \tau .
$$

We apply the same reasoning for $\bar{W}_{n}$ with some positive constant $\epsilon_{2}>0$.

Coming back to (17) it follows that

$$
\begin{aligned}
& \frac{1}{2}\left(\left\|\bar{V}_{n}(T)\right\|_{L^{2}(\Omega)}^{2}+\left\|\bar{W}_{n}(T)\right\|_{L^{2}(\Omega)}^{2}\right) \\
& +\int_{0}^{T} Q(x, \tau)\left(\left\|\bar{V}_{n}(\tau)\right\|_{L^{2}\left(\Gamma_{\text {out }}\right)}^{2}+\left\|\bar{W}_{n}(\tau)\right\|_{L^{2}\left(\Gamma_{\text {out }}\right)}^{2}\right) \mathrm{d} \tau \\
& +\left(D_{\mathrm{S}}-\frac{\|Q\|_{L^{\infty}(\bar{\Omega} \times(0, T))}}{4 \epsilon_{1}}\right) \int_{0}^{T}\left\|\nabla \bar{V}_{n}(\tau)\right\|_{L^{2}(\Omega)}^{2} \mathrm{~d} \tau \\
& +\left(D_{\mathrm{B}}-\frac{\left.\|Q\|_{L^{\infty}(\bar{\Omega} \times(0, T))}\right)}{4 \epsilon_{2}} \int_{0}^{T}\left\|\nabla \bar{W}_{n}(\tau)\right\|_{L^{2}(\Omega)}^{2}\right) \mathrm{d} \tau \\
& +\underbrace{\left(\lambda-\epsilon_{1}\|Q\|_{L^{\infty}(\bar{\Omega} \times(0, T))}-\frac{\|\mu\|_{L^{\infty}(\mathbb{R})}}{2}\right)}_{:=C} \int_{0}^{T}\left\|\bar{V}_{n}(\tau)\right\|_{L^{2}(\Omega)}^{2} \mathrm{~d} \tau \\
& +\left(\lambda-\epsilon_{2}\|Q\|_{L^{\infty}(\bar{\Omega} \times(0, T))}-\frac{3}{2}\|\mu\|_{L^{\infty}(\mathbb{R})}\right) \int_{0}^{T}\left\|\bar{W}_{n}(\tau)\right\|_{L^{2}(\Omega)}^{2} \mathrm{~d} \tau \\
& \leq \int_{0}^{T} \int_{\Omega}\left|\mu(Z(x, \tau))-\mu\left(Z_{n}(x, \tau)\right)\left\|B_{Z_{\mathrm{n}}}(x, \tau)\right\|\left(\bar{W}_{n}(x, \tau)-\bar{V}_{n}(x, \tau)\right)\right| \mathrm{d} x \mathrm{~d} \tau .
\end{aligned}
$$

If $\epsilon_{1}, \epsilon_{2}$ and $\lambda$ are chosen such that $\epsilon_{1} \geq \frac{\|Q\|_{L^{\infty}(\bar{\Omega} \times(0, T))}}{4 D_{\mathrm{S}}}, \epsilon_{2} \geq \frac{\|Q\|_{L^{\infty}(\bar{\Omega} \times(0, T))}}{4 D_{\mathrm{B}}}$ and $\lambda>$ $\|Q\|_{L^{\infty}(\bar{\Omega} \times(0, T))} \max \left(\epsilon_{1}, \epsilon_{2}\right)+\frac{3}{2}\|\mu\|_{L^{\infty}(\mathbb{R})}$, one has

$$
\begin{aligned}
& \int_{0}^{T}\left\|\bar{V}_{n}(\tau)\right\|_{L^{2}(\Omega)}^{2} \mathrm{~d} \tau \\
& \quad \leq 2 \int_{0}^{T} \int_{\Omega}\left|\mu(Z(x, \tau))-\mu\left(Z_{\mathrm{n}}(x, \tau)\right)\left\|B_{\mathrm{Z}_{\mathrm{n}}}(x, \tau)\right\|\left(\bar{W}_{n}(x, \tau)-\bar{V}_{n}(x, \tau)\right)\right| \mathrm{d} x \mathrm{~d} \tau .
\end{aligned}
$$

To prove that the right hand side of (19) converges to 0 as $n \rightarrow \infty$, we use the following steps: 
1. Since $\left\|Z_{n}-Z\right\|_{L^{2}(\Omega \times(0, T))} \stackrel{n \rightarrow \infty}{\longrightarrow} 0$, using Theorem A.15, there exists a subsequence $\left\{Z_{n_{k}}\right\}_{k} \subset\left\{Z_{n}\right\}_{n}$ such that $Z_{\mathrm{n}_{\mathrm{k}}} \rightarrow Z$ a.e. in $\Omega \times(0, T)$. Then, since $\mu$ is continuous, $\mu\left(Z_{\mathrm{n}_{\mathrm{k}}}\right) \rightarrow \mu(Z)$ a.e. in $\Omega \times(0, T)$. For simplicity, we denote $\left\{Z_{n_{k}}\right\}_{k}=\left\{Z_{k}\right\}_{k}$.

2. Since $\left\|\mu\left(Z_{k}\right)\right\|_{L^{\infty}(Q)} \leq\|\mu\|_{L^{\infty}(\mathbb{R})}<+\infty$, by applying Theorem $\mathbf{A . 1 4}$ (using that $L^{1}(\Omega \times$ $(0, T))$ is separable and $\left.\left(L^{1}(\Omega \times(0, T))\right)^{\prime}=L^{\infty}(\Omega \times(0, T))\right)$, there exists a subsequence $\left\{\mu\left(Z_{k_{j}}\right)\right\}_{j}$ weak-* convergent to some $k \in L^{\infty}(\Omega \times(0, T))$. For simplicity, we denote $\left\{Z_{k_{j}}\right\}_{j}=\left\{Z_{j}\right\}_{j}$.

Due to steps 1 and 2 , we conclude that $\left\{\mu\left(Z_{\mathrm{j}}\right)\right\}_{j}$ is weak-* convergent to $\mu(Z)$.

3. $B_{\mathrm{Z}_{\mathrm{j}}} \in K$, since $\left(S_{\mathrm{Z}_{\mathrm{j}}}, B_{\mathrm{Z}_{\mathrm{j}}}\right)$ is solution of (3) with $c=\mu\left(Z_{j}\right)$. Moreover, since $K \subset X$ is compact, there exists a subsequence $\left\{B_{Z_{j_{i}}}\right\}_{i} \subset\left\{B_{\mathrm{Z}_{\mathrm{j}}}\right\}_{j}$ such that there exist some $B \in X$ fulfilling $\left\|B_{\mathrm{Z}_{\mathrm{j}_{\mathrm{i}}}}-B\right\|_{X} \stackrel{i \rightarrow \infty}{\longrightarrow} 0$. For simplicity, we denote $\left\{Z_{j_{i}}\right\}_{i}=\left\{Z_{i}\right\}_{i}$.

4. We define

$$
\bar{K}=\left\{z \in W\left(0, T, H^{1}(\Omega),\left(H^{1}(\Omega)\right)^{\prime}\right):\|z\|_{W\left(0, T, H^{1}(\Omega),\left(H^{1}(\Omega)\right)^{\prime}\right)} \leq 4 \bar{C}\right\},
$$

where $\bar{C}$ is the constant appearing in the definition of $K$ in (13). Notice that $\bar{K}$ is a compact set of $X$ (see Remark A.7 and Definition A.5). Since $W_{i}-V_{i}=B_{Z_{\mathrm{i}}}-S_{Z_{\mathrm{i}}}-B_{\mathrm{Z}}+$ $S_{\mathrm{Z}} \in \bar{K}$, using the same reasoning as the one followed above, one obtains that there exists a subsequence $\left\{W_{i_{r}}-V_{i_{r}}\right\}_{r} \subset\left\{W_{i}-V_{i}\right\}_{i}$ and $P \in X$ such that $\left\|\left(W_{i_{r}}-V_{i_{r}}\right)-P\right\|_{X} \stackrel{r \rightarrow \infty}{\longrightarrow} 0$. For simplicity, we denote $\left\{Z_{i_{r}}\right\}_{r}=\left\{Z_{r}\right\}_{r}$.

By steps 3 and 4 , we conclude that $B_{\mathrm{r}}\left(W_{r}-V_{r}\right) \subset L^{1}(\Omega \times(0, T))$ and $\| B_{\mathrm{r}}\left(W_{r}-V_{r}\right)-$ $B P \|_{L^{1}(\Omega \times(0, T))} \stackrel{r \rightarrow \infty}{\longrightarrow} 0$.

Furthermore, since $\left\{Z_{r}\right\}_{r} \subset\left\{Z_{j}\right\}_{j}$, it also follows that $\left\{\mu\left(Z_{\mathrm{r}}\right)\right\}_{r}$ is weak-* convergent to $\mu(Z)$. Using Theorem A.16, if follows that

$$
\begin{gathered}
\int_{0}^{T} \int_{\Omega} \underbrace{\left|\mu(Z(x, \tau))-\mu\left(Z_{\mathrm{r}}(x, \tau)\right)\right|}_{L^{\infty}(Q)} \underbrace{\left|B_{\mathrm{Z}_{\mathrm{r}}}(x, \tau)\right|\left|\left(\bar{W}_{r}(x, \tau)-\bar{V}_{r}(x, \tau)\right)\right|}_{L^{1}(Q)} \mathrm{d} x \mathrm{~d} \tau \\
\stackrel{r \rightarrow \infty}{\longrightarrow} \int_{0}^{T} \int_{\Omega} 0 \cdot B(x, \tau) \cdot P(x, \tau) \mathrm{d} \tau \mathrm{d} x .
\end{gathered}
$$

From (19), this implies that

$$
\int_{0}^{T} \int_{\Omega} e^{-2 \lambda \tau}\left|S_{\mathrm{Z}_{\mathrm{r}}}(x, \tau)-S_{\mathrm{Z}}(x, \tau)\right|^{2} \mathrm{~d} x \mathrm{~d} \tau \stackrel{r \rightarrow \infty}{\longrightarrow} 0
$$

but since $\min _{\tau \in[0, T]} e^{-2 \lambda \tau}=e^{-2 \lambda T}$, one has that

$$
\left\|S_{\mathrm{Z}_{\mathrm{r}}}-S_{Z}\right\|_{L^{2}(\Omega \times(0, T))} \stackrel{r \rightarrow \infty}{\longrightarrow} 0 .
$$

Finally, we prove that $\left\|S_{Z_{n}}-S_{Z}\right\|_{L^{2}(\Omega \times(0, T))} \stackrel{n \rightarrow \infty}{\longrightarrow} 0$ (convergence of the whole sequence instead of subsequence) by reduction to absurdum. Let us assume that this is not true. Then, there exists $\epsilon>0$ and a subsequence $\left\{S_{\mathrm{Z}_{\mathrm{n}_{1}}}\right\}_{l} \subset\left\{S_{\mathrm{Z}_{\mathrm{n}}}\right\}_{n}$ such that

$$
\left\|S_{\mathrm{Z}_{\mathrm{n}_{1}}}-S_{Z}\right\|_{L^{2}(\Omega \times(0, T))}>\epsilon \quad, \forall l \in \mathbb{N} .
$$

If we know proceed as above, we can find a subsection $\left\{S_{\mathrm{Z}_{\mathrm{n}_{\mathrm{m}}}}\right\}_{m} \subset\left\{S_{\mathrm{Z}_{\mathrm{n}_{1}}}\right\}_{l}$ such that

$$
\left\|S_{\mathrm{Z}_{\mathrm{n}_{\mathrm{m}}}}-S_{Z}\right\|_{L^{2}(\Omega \times(0, T))} \stackrel{m \rightarrow \infty}{\longrightarrow} 0,
$$

which contradicts (21). 
Now, we are interested in studying the nonnegativity and boundedness properties of solutions $B$ and $S$.

Theorem 3.6 (Nonnegativity and boundedness of B). Under assumptions of Theorem [3.1:

(i) If $B_{\text {init }} \geq 0$ in $\Omega$, then $B \geq 0$ in $\Omega \times(0, T)$.

(ii) If $B_{\text {init }} \in L^{\infty}(\Omega)$, then $B(x, t) \leq\left\|B_{\text {init }}\right\|_{L^{\infty}(\Omega)} e^{\|\mu\|_{L^{\infty}(\mathrm{R})} t}$ a.e. $(x, t) \in \Omega \times(0, T)$.

Proof. Function $B$ satisfies the following system:

$$
\left\{\begin{array}{lr}
B_{\mathrm{t}}=\operatorname{div}\left(D_{\mathrm{B}} \nabla B-\mathbf{Q} B\right)+\mu(S) B & \text { in } \Omega \times(0, T), \\
B(x, 0)=B_{\text {init }}(x) & \text { in } \Omega, \\
\mathbf{n} \cdot\left(-D_{\mathrm{B}} \nabla B+\mathbf{Q} B\right)=0 & \text { in } \Gamma_{\text {in }} \cup \Gamma_{\text {wall }} \times(0, T), \\
\mathbf{n} \cdot\left(-D_{\mathrm{B}} \nabla B\right)=0 & \text { in } \Gamma_{\text {out }} \times(0, T) .
\end{array}\right.
$$

If we define the new variables $B^{+}=\max (B, 0)$ and $B^{-}=-\min (B, 0)$, then $B=B^{+}-B^{-}$ and the first statement of Theorem 3.6 can be reformulated as

$$
B^{-}(x, 0)=0 \text { in } \Omega \Rightarrow B^{-}(x, t)=0 \quad \text { in } \Omega \times(0, T) .
$$

Multiplying the first equation of (22) by $B^{-}$and integrating, one obtains

$\frac{1}{2} \int_{0}^{t} \frac{\mathrm{d}}{\mathrm{d} \tau}\left\|B^{-}(\tau)\right\|_{\mathrm{L}^{2}(\Omega)}^{2} \mathrm{~d} \tau=\int_{0}^{t} \int_{\Omega} \mathbf{Q}(x, \tau) B^{-}(x, \tau) \nabla B^{-}(x, \tau) \mathrm{d} x \mathrm{~d} \tau$

$-\int_{0}^{t} \int_{\Gamma_{\text {out }}} Q(x, \tau)\left(B^{-}(x, \tau)\right)^{2} \mathrm{~d} x \mathrm{~d} \tau-\int_{0}^{t} \int_{\Omega} D_{\mathrm{B}}\left(\nabla B^{-}(x, \tau)\right)^{2} \mathrm{~d} x \mathrm{~d} \tau$

$+\int_{0}^{t} \int_{\Omega} \mu(S(x, \tau)) B^{-}(x, \tau)^{2} \mathrm{~d} x \mathrm{~d} \tau$.

Applying Young's inequality with $\epsilon>0$ (that will be specified below), one has:

$$
\begin{aligned}
\frac{1}{2} \int_{0}^{t} \frac{\mathrm{d}}{\mathrm{d} \tau}\left\|B^{-}(\tau)\right\|_{\mathrm{L}^{2}(\Omega)}^{2} \mathrm{~d} \tau \leq & \left(\epsilon\|Q\|_{L^{\infty}(\bar{\Omega} \times(0, T))}-D_{\mathrm{B}}\right) \int_{0}^{t}\left\|\nabla B^{-}(\tau)\right\|_{L^{2}(\Omega)}^{2} \mathrm{~d} \tau \\
& +\left(\frac{\|Q\|_{L^{\infty}(\bar{\Omega} \times(0, T))}}{4 \epsilon}+\|\mu\|_{L^{\infty}(\mathbb{R})}\right) \int_{0}^{T}\left\|B^{-}(\tau)\right\|_{L^{2}(\Omega)}^{2} \mathrm{~d} \tau .
\end{aligned}
$$

Choosing $\epsilon$ such that $\epsilon\|Q\|_{L^{\infty}(\bar{\Omega} \times(0, T))}-D_{\mathrm{B}} \leq 0$ and applying Gronwall's Inequality in its integral form (see Theorem A.11), one has:

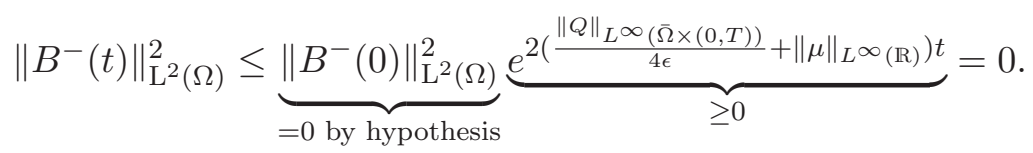

Consequently $B^{-}=0$ in $\Omega \times(0, T)$ and the statement (i) of the theorem is proved.

Now, we define $U(x, t)=\left\|B_{\text {init }}\right\|_{L^{\infty}(\Omega)} e^{\|\mu\|_{L^{\infty}(\mathbb{R})} t}-B(x, t)$. We want to prove that $U(x, t) \geq$ 0 in $\Omega \times(0, T)$. It fulfills 


$$
\left\{\begin{array}{lr}
U_{\mathrm{t}}=\operatorname{div}\left(D_{\mathrm{B}} \nabla U-\mathbf{Q} U\right)+\mu(S) U+\alpha e^{\|\mu\|_{L^{\infty}(\mathbb{R})} t} & \text { in } \Omega \times(0, T), \\
U(x, 0)=\left\|B_{\text {init }}\right\|_{L^{\infty}(\Omega)}-B_{\text {init }}(x) & \text { in } \Omega, \\
\mathbf{n} \cdot\left(D_{\mathrm{B}} \nabla U-\mathbf{Q} U\right)=Q(t)\left\|B_{\text {init }}\right\|_{L^{\infty}(\Omega)} e^{\|\mu\|_{L^{\infty}(\mathbb{R})} t} & \text { in } \Gamma_{\text {in }} \times(0, T), \\
\mathbf{n} \cdot\left(D_{\mathrm{B}} \nabla U-\mathbf{Q} U\right)=0 & \text { in } \Gamma_{\text {wall }} \times(0, T), \\
\mathbf{n} \cdot\left(-D_{\mathrm{B}} \nabla U\right)=0 & \text { in } \Gamma_{\text {out }} \times(0, T),
\end{array}\right.
$$

where $\alpha=\left(\|\mu\|_{L^{\infty}(\mathbb{R})}-\mu(S)\right)\left\|B_{\text {init }}\right\|_{L^{\infty}(\Omega)}$.

We define the new variables $U^{+}=\max (U, 0)$ and $U^{-}=-\min (U, 0)$. Multiplying the first equation of (23) by $U^{-}$and integrating, we have

$\frac{1}{2} \int_{0}^{t} \frac{\mathrm{d}}{\mathrm{d} \tau}\left\|U^{-}(\tau)\right\|_{\mathrm{L}^{2}(\Omega)}^{2} \mathrm{~d} \tau=-\int_{0}^{t} \int_{\Gamma_{\text {out }}} Q(x, \tau)\left(U^{-}(x, \tau)\right)^{2} \mathrm{~d} x \mathrm{~d} \tau$

$-\int_{0}^{t} \int_{\Gamma_{\text {in }}} Q(x, \tau)\left\|B_{\text {init }}\right\|_{L^{\infty}(\Omega)} e^{\|\mu\|_{L^{\infty}(\mathbb{R})} \tau} U^{-}(x, \tau) \mathrm{d} x \mathrm{~d} \tau-\int_{0}^{t} \int_{\Omega} D_{\mathrm{B}}\left(\nabla U^{-}(x, \tau)\right)^{2} \mathrm{~d} x \mathrm{~d} \tau$

$+\int_{0}^{t} \int_{\Omega} \mathbf{Q}(x, \tau) U^{-}(x, \tau) \nabla U^{-}(x, \tau) \mathrm{d} x \mathrm{~d} \tau+\int_{0}^{t} \int_{\Omega} \mu(S(x, \tau))\left(U^{-}(x, \tau)\right)^{2} \mathrm{~d} x \mathrm{~d} \tau$

$+\int_{0}^{t} \int_{\Omega} \alpha e^{\|\mu\|_{L} \infty(\Omega)}{ }^{t}\left(-U^{-}(x, \tau)\right) \mathrm{d} x \mathrm{~d} \tau$.

Since $Q$ and $U^{-}$are nonnegative, applying Young's inequality with $\epsilon>0$ (that we will choose below), it follows

$$
\begin{aligned}
\frac{1}{2} \int_{0}^{t} \frac{\mathrm{d}}{\mathrm{d} \tau}\left\|U^{-}(\tau)\right\|_{\mathrm{L}^{2}(\Omega)}^{2} \mathrm{~d} \tau \leq & \left(\epsilon\|Q\|_{L^{\infty}(\bar{\Omega} \times(0, T))}-D_{\mathrm{B}}\right) \int_{0}^{T}\left\|\nabla U^{-}(\tau)\right\|_{L^{2}(\Omega)}^{2} \mathrm{~d} \tau \\
& +\left(\|\mu\|_{L^{\infty}(\Omega)}+\frac{\|Q\|_{L^{\infty}(\bar{\Omega} \times(0, T))}}{4 \epsilon} \int_{0}^{T}\left\|U^{-}(\tau)\right\|_{L^{2}(\Omega)}^{2} \mathrm{~d} \tau\right.
\end{aligned}
$$

Choosing $\epsilon$ such that $\epsilon\|Q\|_{L^{\infty}(\bar{\Omega} \times(0, T))}-D_{\mathrm{B}} \leq 0$ and applying Gronwall's Inequality in its integral form (see Theorem A.11), one has:

$$
\left\|U^{-}(t)\right\|_{\mathrm{L}^{2}(\Omega)}^{2} \leq\left\|U^{-}(0)\right\|_{L^{2}(\Omega)}^{2} e^{2\left(\|\mu\|_{L^{\infty}(\Omega)}+\frac{\left.\|Q\|_{L^{\infty}(\bar{\Omega} \times(0, T))}\right) t}{4 \epsilon} .\right.}
$$

Since $U(x, 0) \geq 0$, then $\left\|U^{-}(0)\right\|_{\mathrm{L}^{2}(\Omega)}^{2}=0$ and, consequently, $U^{-}=0$ in $\Omega \times(0, T)$ and the statement (ii) of the theorem is proved.

Theorem 3.7 (Nonnegativity and boundedness of $S$ ). Under assumptions of Theorems 3.1 and 3.6-(ii), if $S_{\mathrm{e}} \geq 0$ and $S_{\mathrm{init}} \geq 0$ in $\Omega, \mu$ is lipschitz and $\mu(0)=0$, then $S \geq 0$ in $\Omega \times(0, T)$. Furthermore, if $S_{\text {init }} \in L^{\infty}(\Omega), S_{\mathrm{e}} \in L^{\infty}(0, T)$ and $\mu(z)>0$ for $z>0$, then $S \leq \max \left(\left\|S_{\text {init }}\right\|_{\mathrm{L}^{\infty}(\Omega)},\left\|S_{\mathrm{e}}\right\|_{\mathrm{L}^{\infty}(0, T)}\right)$ in $\Omega \times(0, T)$. 
Proof. Function $S$ satisfies the following system:

$$
\left\{\begin{array}{lr}
S_{\mathrm{t}}=\operatorname{div}\left(D_{\mathrm{S}} \nabla S-\mathbf{Q} S\right)-\mu(S) B & \text { in } \Omega \times(0, T), \\
S(x, 0)=S_{\text {init }}(x) & \text { in } \Omega, \\
\mathbf{n} \cdot\left(D_{\mathrm{S}} \nabla S-\mathbf{Q} S\right)=Q S_{\mathrm{e}} & \text { in } \Gamma_{\text {in }} \times(0, T), \\
\mathbf{n} \cdot\left(D_{\mathrm{S}} \nabla S-\mathbf{Q} S\right)=0 & \text { in } \Gamma_{\text {wall }} \times(0, T), \\
\mathbf{n} \cdot\left(D_{\mathrm{S}} \nabla S\right)=0 & \text { in } \Gamma_{\text {out }} \times(0, T) .
\end{array}\right.
$$

If we define the new variables $S^{+}=\max (S, 0)$ and $S^{-}=-\min (S, 0)$, then $S=S^{+}-S^{-}$ and multiplying the first equation of (24) by $S^{-}$and integrating it follows

$$
\begin{aligned}
& \frac{1}{2} \int_{0}^{t} \frac{\mathrm{d}}{\mathrm{d} \tau}\left\|S^{-}(\tau)\right\|_{\mathrm{L}^{2}(\Omega)}^{2} \mathrm{~d} \tau=-\int_{0}^{t} \int_{\Gamma_{\text {out }}} Q(x, \tau)\left(S^{-}(x, \tau)\right)^{2} \mathrm{~d} x \mathrm{~d} \tau \\
& -\int_{0}^{t} \int_{\Gamma_{\text {in }}} Q(x, \tau) S_{\mathrm{e}}(\tau) S^{-}(x, \tau) \mathrm{d} x \mathrm{~d} \tau-\int_{0}^{t} \int_{\Omega} D_{\mathrm{S}}\left(\nabla S^{-}(x, \tau)\right)^{2} \mathrm{~d} x \mathrm{~d} \tau \\
& +\int_{0}^{t} \int_{\Omega} \mathbf{Q}(x, \tau) S^{-}(x, \tau) \nabla S^{-}(x, \tau) \mathrm{d} x \mathrm{~d} \tau \\
& +\int_{0}^{t} \int_{\Omega} \mu(S(x, \tau)) B(x, \tau) S^{-}(x, \tau) \mathrm{d} x \mathrm{~d} \tau .
\end{aligned}
$$

Under the hypothesis formulated on $\mu$, there exists a constant $C_{\mathrm{L}}$ such that

$\left|\int_{0}^{t} \int_{\Omega} \mu(S(x, \tau)) B(x, \tau) S^{-}(x, \tau) \mathrm{d} x \mathrm{~d} \tau\right|$

$\leq C_{\mathrm{L}} \int_{0}^{t} \int_{\Omega}|S(x, \tau)||B(x, \tau)| S^{-}(x, \tau) \mathrm{d} x \mathrm{~d} \tau$

$\leq C_{\mathrm{L}}\|B\|_{L^{\infty}(\Omega \times(0, T))} \int_{0}^{t} \int_{\Omega}\left(S^{-}(x, \tau)\right)^{2} \mathrm{~d} x \mathrm{~d} \tau$.

Furthermore, since $S_{\mathrm{e}}, Q$ and $S^{-}$are nonnegative, coming back to equation (25), one obtains

$$
\begin{aligned}
\frac{1}{2} \int_{0}^{t} \frac{\mathrm{d}}{\mathrm{d} \tau}\left\|S^{-}(\tau)\right\|_{\mathrm{L}^{2}(\Omega)}^{2} \mathrm{~d} \tau & \leq-\int_{0}^{t} \int_{\Omega} D_{\mathrm{S}}\left(\nabla S^{-}(x, \tau)\right)^{2} \mathrm{~d} x \mathrm{~d} \tau \\
& +\int_{0}^{t} \int_{\Omega} \mathbf{Q}(x, \tau) S^{-}(x, \tau) \nabla S^{-}(x, \tau) \mathrm{d} x \mathrm{~d} \tau \\
& +C_{\mathrm{L}}\|B\|_{L^{\infty}(\Omega \times(0, T))} \int_{0}^{t} \int_{\Omega}\left(S^{-}(x, \tau)\right)^{2} \mathrm{~d} x \mathrm{~d} \tau .
\end{aligned}
$$

Moreover, applying Young's inequality with $\epsilon>0$ (that will be specified below), one has:

$$
\begin{aligned}
& \frac{1}{2} \int_{0}^{t} \frac{\mathrm{d}}{\mathrm{d} \tau}\left\|S^{-}(\tau)\right\|_{\mathrm{L}^{2}(\Omega)}^{2} \mathrm{~d} \tau \leq\left(\epsilon\|Q\|_{L^{\infty}(\bar{\Omega} \times(0, T))}-D_{\mathrm{S}}\right) \int_{0}^{T}\left\|\nabla S^{-}(\tau)\right\|_{L^{2}(\Omega)}^{2} \mathrm{~d} \tau \\
&+\left(\frac{\|Q\|_{L^{\infty}(\bar{\Omega} \times(0, T))}}{4 \epsilon}+C_{\mathrm{L}}\|B\|_{L^{\infty}(\Omega \times(0, T))}\right) \int_{0}^{T}\left\|S^{-}(\tau)\right\|_{L^{2}(\Omega)}^{2} \mathrm{~d} \tau
\end{aligned}
$$

Choosing $\epsilon$ such that $\epsilon\|Q\|_{L^{\infty}(\bar{\Omega} \times(0, T))}-D_{\mathrm{S}} \leq 0$ and applying Gronwall's Inequality in its integral form (see Theorem A.11), one has: 


$$
\left\|S^{-}(t)\right\|_{\mathrm{L}^{2}(\Omega)}^{2} \leq \underbrace{\left\|S^{-}(0)\right\|_{\mathrm{L}^{2}(\Omega)}^{2}}_{=0 \text { by hypothesis }} e^{2\left(\frac{\|Q\|_{L^{\infty}(\bar{\Omega} \times(0, T))}}{4 \epsilon}+C_{\mathrm{L}}\|B\|_{L^{\infty}(\Omega \times(0, T))}\right) t}=0 .
$$

Consequently $S^{-}=0$ in $\Omega \times(0, T)$ and the first statement of the theorem is proved.

Now, we define $\beta=\max \left(\left\|S_{\text {init }}\right\|_{L^{\infty}(\Omega)},\left\|S_{\mathrm{e}}\right\|_{L^{\infty}(0, T)}\right)$ and $U(x, t)=\beta-S(x, t)$. We want to prove that $U(x, t) \geq 0$ in $\Omega \times(0, T)$. It fulfills

$$
\left\{\begin{array}{lr}
U_{\mathrm{t}}=\operatorname{div}\left(D_{\mathrm{S}} \nabla U-\mathbf{Q} U\right)+\mu(S) B & \text { in } \Omega \times(0, T), \\
U(x, 0)=\beta-S_{\text {init }}(x) & \text { in } \Omega, \\
\mathbf{n} \cdot\left(D_{\mathrm{S}} \nabla U-\mathbf{Q} U\right)=Q\left(\beta-S_{\mathrm{e}}\right) & \text { in } \Gamma_{\text {in }} \times(0, T), \\
\mathbf{n} \cdot\left(D_{\mathrm{S}} \nabla U-\mathbf{Q} U\right)=0 & \text { in } \Gamma_{\text {wall }} \times(0, T), \\
\mathbf{n} \cdot\left(D_{\mathrm{S}} \nabla U\right)=0 & \text { in } \Gamma_{\text {out }} \times(0, T) .
\end{array}\right.
$$

We define the new variables $U^{+}=\max (U, 0)$ and $U^{-}=-\min (U, 0)$. Multiplying the first equation of (27) by $U^{-}$and integrating, it follows

$\left.\frac{1}{2} \int_{0}^{t} \frac{\mathrm{d}}{\mathrm{d} \tau}\left\|U^{-}(\tau)\right\|_{\mathrm{L}^{2}(\Omega)}^{2} \mathrm{~d} \tau=-\int_{0}^{t} \int_{\Gamma_{\text {out }}} Q(x, \tau)\left(U^{-}(x, \tau)\right)\right)^{2} \mathrm{~d} x \mathrm{~d} \tau$

$-\int_{0}^{t} \int_{\Gamma_{\mathrm{in}}} Q(x, \tau)\left(\beta-S_{\mathrm{e}}(\tau)\right) U^{-}(x, \tau) \mathrm{d} x \mathrm{~d} \tau-\int_{0}^{t} \int_{\Omega} D_{\mathrm{S}}\left(\nabla U^{-}(x, \tau)\right)^{2} \mathrm{~d} x \mathrm{~d} \tau$

$+\int_{0}^{t} \int_{\Omega} \mathbf{Q}(x, \tau) U^{-}(x, \tau) \nabla U^{-}(x, \tau) \mathrm{d} x \mathrm{~d} \tau-\int_{0}^{t} \int_{\Omega} \mu(S(x, \tau)) B(x, \tau) U^{-}(x, \tau) \mathrm{d} x \mathrm{~d} \tau$.

Since $S(x, t) \geq 0$ in $\Omega \times(0, T)$, by the hypothesis formulated on $\mu$ one has that $\mu(S(x, t)) \geq$ 0 . Furthermore, taking into account that $Q, B$ and $U^{-}$are nonnegative (see Theorem 3.6), applying Young's inequality 36 with $\epsilon>0$ (chosen below), one has

$$
\begin{aligned}
\frac{1}{2} \int_{0}^{t} \frac{\mathrm{d}}{\mathrm{d} \tau}\left\|U^{-}(\tau)\right\|_{\mathrm{L}^{2}(\Omega)}^{2} \mathrm{~d} \tau \leq & \left(\epsilon\|Q\|_{L^{\infty}(\bar{\Omega} \times(0, T))}-D_{\mathrm{S}}\right) \int_{0}^{T}\left\|\nabla U^{-}(\tau)\right\|_{L^{2}(\Omega)}^{2} \mathrm{~d} \tau \\
& +\frac{\|Q\|_{L^{\infty}(\bar{\Omega} \times(0, T))}}{4 \epsilon} \int_{0}^{T}\left\|U^{-}(\tau)\right\|_{L^{2}(\Omega)}^{2} \mathrm{~d} \tau .
\end{aligned}
$$

Choosing $\epsilon$ such that $\epsilon\|Q\|_{L^{\infty}(\bar{\Omega} \times(0, T))}-D_{\mathrm{B}} \leq 0$ and applying Gronwall's Inequality in its integral form (see Theorem A.11), one obtains

$$
\left\|U^{-}(t)\right\|_{L^{2}(\Omega)}^{2} \leq\left\|U^{-}(0)\right\|_{L^{2}(\Omega)}^{2} e^{\frac{\|Q\|_{L^{\infty}(\bar{\Omega} \times(0, T))}}{2 \epsilon} t} .
$$

Since $U(x, 0) \geq 0$, then $\left\|U^{-}(0)\right\|_{L^{2}(\Omega)}^{2}=0$ and, consequently, $U^{-}=0$ in $\Omega \times(0, T)$ and the second statement of the theorem is proved.

Remark 3.8. Notice that we assume that $Q, S_{\mathrm{e}}, B_{\text {init }}$ and $S_{\text {init }}$ are nonnegative and essentially bounded because of their physical meaning. The assumption $\mu(0)=0$ is due to the fact that if there is no substrate concentration, no reaction is produced; the assumption $\mu(z)>0$ if $z>0$ follows from the fact that if there is substrate, the reaction makes the substrate concentration decrease and the biomass concentration increase (see System (1)). These two assumptions, together with the hypothesis that $\mu$ is an increasing function (assumed in Theorem (3.9) are 
commonly used in bioreactor theory (see e.g, 24]). Furthermore, the assumption of considering that function $\mu$ is essentially bounded is a caused by the fact that microorganisms have a maximum specific growth rate.

Finally, we prove the uniqueness of solution of System (10).

Theorem 3.9 (Uniqueness of solution). Under the hypothesis of Theorem [3.6 and if $\mu$ is increasing and Lipschitz, then System (1) has a unique weak solution $(S, B)$.

Proof. Let us assume that $\left(S_{1}, B_{1}\right)$ and $\left(S_{2}, B_{2}\right)$ are two different weak solutions of System (11). We denote $V=S_{1}-S_{2}, W=B_{1}-B_{2}$ and $\bar{V}=e^{-\lambda t} V, \bar{W}=e^{-\lambda t} W$, where $\lambda>0$ will be chosen later. Proceeding as in previous theorems, we can obtain the following energy estimate:

$$
\begin{aligned}
& \frac{1}{2}\|\bar{V}(T)\|_{L^{2}(\Omega)}^{2}+\lambda \int_{0}^{T}\|\bar{V}(\tau)\|_{L^{2}(\Omega)}^{2} \mathrm{~d} \tau+\int_{0}^{T} \int_{\Gamma_{\text {out }}} Q(x, \tau) \bar{V}(x, \tau)^{2} \mathrm{~d} x \mathrm{~d} \tau \\
& +D_{\mathrm{S}} \int_{0}^{T}\|\nabla \bar{V}(\tau)\|_{L^{2}(\Omega)}^{2} \mathrm{~d} \tau=\int_{0}^{T} \int_{\Omega} \mathbf{Q} \bar{V}(x, \tau) \nabla \bar{V}(x, \tau) \mathrm{d} x \mathrm{~d} \tau \\
& +\underbrace{\int_{0}^{T} e^{-\lambda \tau} \int_{\Omega}\left(\mu\left(S_{2}(x, \tau)\right) B_{2}(x, \tau)-\mu\left(S_{1}(x, \tau)\right) B_{1}(x, \tau)\right) \bar{V}(x, \tau) \mathrm{d} x \mathrm{~d} \tau}_{(I)} .
\end{aligned}
$$

Now,

$$
\begin{aligned}
(I)=\int_{0}^{T} e^{-\lambda \tau} \int_{\Omega} \mu\left(S_{1}(x, \tau)\right)\left(B_{2}(x, \tau)-B_{1}(x, \tau)\right) \bar{V}(x, \tau) \mathrm{d} x \mathrm{~d} \tau \\
\quad+\int_{0}^{T} e^{-\lambda \tau} \int_{\Omega}\left(\mu\left(S_{2}(x, \tau)\right)-\mu\left(S_{1}(x, \tau)\right)\right) B_{2}(x, \tau) \bar{V}(x, \tau) \mathrm{d} x \mathrm{~d} \tau .
\end{aligned}
$$

Since $\mu$ is increasing, $\left(\mu\left(S_{2}(x, \tau)\right)-\mu\left(S_{1}(x, \tau)\right)\right)\left(S_{1}(x, \tau)-S_{2}(x, \tau)\right) \leq 0$. Moreover, by Theorem 3.6, it follows that $B_{2} \geq 0$ in $\Omega \times(0, T)$, and

$$
\begin{aligned}
(I) & \leq\|\mu\|_{L^{\infty}(\mathbb{R})} \int_{0}^{T} \int_{\Omega}|\bar{V}(x, \tau) \| \bar{W}(x, \tau)| \mathrm{d} x \mathrm{~d} \tau \\
& \leq \frac{\|\mu\|_{L^{\infty}(\mathbb{R})}}{2} \int_{0}^{T}\left(\|\bar{V}(\tau)\|_{L^{2}(\Omega)}^{2}+\|\bar{W}(\tau)\|_{L^{2}(\Omega)}^{2}\right) \mathrm{d} \tau .
\end{aligned}
$$

Coming back to (28) and applying Holder's and Young's inequality (36) with $\epsilon_{1}>0$ (that will be chosen later), one has:

$$
\begin{aligned}
& \frac{1}{2}\|\bar{V}(T)\|_{L^{2}(\Omega)}^{2}+\left(\lambda-\epsilon_{1}\|Q\|_{L^{\infty}(\bar{\Omega} \times(0, T))}-\frac{\|\mu\|_{L^{\infty}(\mathbb{R})}}{2}\right) \int_{0}^{T}\|\bar{V}(\tau)\|_{L^{2}(\Omega)}^{2} \mathrm{~d} \tau \\
& +\int_{0}^{T} \int_{\Gamma_{\text {out }}} Q(x, \tau) \bar{V}(x, \tau)^{2} \mathrm{~d} x \mathrm{~d} \tau+\left(D_{\mathrm{S}}-\frac{\|Q\|_{L^{\infty}(\bar{\Omega} \times(0, T))}}{4 \epsilon_{1}}\right) \int_{0}^{T}\|\nabla \bar{V}(\tau)\|_{L^{2}(\Omega)}^{2} \mathrm{~d} \tau \\
& \leq \frac{\|\mu\|_{L^{\infty}(\mathrm{R})}}{2} \int_{0}^{T}\|\bar{W}(\tau)\|_{L^{2}(\Omega)}^{2} \mathrm{~d} \tau .
\end{aligned}
$$


Proceeding analogously, we obtain the following energy estimate

$$
\begin{aligned}
& \frac{1}{2}\|\bar{W}(T)\|_{L^{2}(\Omega)}^{2}+\lambda \int_{0}^{T}\|\bar{W}(\tau)\|_{L^{2}(\Omega)}^{2} \mathrm{~d} \tau+\int_{0}^{T} \int_{\Gamma_{\text {out }}} Q(x, \tau) \bar{W}(x, \tau)^{2} \mathrm{~d} x \mathrm{~d} \tau \\
& +D_{\mathrm{B}} \int_{0}^{T}\|\nabla \bar{W}(\tau)\|_{L^{2}(\Omega)}^{2} \mathrm{~d} \tau=\int_{0}^{T} \int_{\Omega} \mathbf{Q} \bar{W}(x, \tau) \nabla \bar{W}(x, \tau) \mathrm{d} x \mathrm{~d} \tau \\
& +\underbrace{\int_{0}^{T} e^{-\lambda \tau} \int_{\Omega}\left(\mu\left(S_{1}(x, \tau)\right) B_{1}(x, \tau)-\mu\left(S_{2}(x, \tau)\right) B_{2}(x, \tau)\right) \bar{W}(x, \tau) \mathrm{d} x \mathrm{~d} \tau}_{(I I)} .
\end{aligned}
$$

Now,

$$
\begin{aligned}
(I I)= & \int_{0}^{T} \int_{\Omega} \mu\left(S_{1}(x, \tau)\right) \bar{W}(x, \tau)^{2} \mathrm{~d} x \mathrm{~d} \tau \\
& +\int_{0}^{T} e^{-\lambda \tau} \int_{\Omega}\left(\mu\left(S_{1}(x, \tau)\right)-\mu\left(S_{2}(x, \tau)\right)\right) B_{2}(x, \tau) \bar{W}(x, \tau) \mathrm{d} x \mathrm{~d} \tau
\end{aligned}
$$

Using the fact that $\mu$ is Lipschitz, there exist some constant $C_{L}>0$ such that $(I I) \leq\|\mu\|_{L^{\infty}(\mathbb{R})} \int_{0}^{T}\|\bar{W}(\tau)\|_{L^{2}(\Omega)}^{2} \mathrm{~d} \tau+C_{L} \int_{0}^{T} \int_{\Omega}\left|\bar{W}(x, \tau) \bar{V}(x, \tau) B_{2}(x, \tau)\right| \mathrm{d} x \mathrm{~d} \tau$.

Since $B_{2} \in L^{\infty}(\Omega \times(0, T))$ (see Theorem 3.6$)$ ), applying Young's inequality (36) with $\epsilon=\frac{1}{2}$, one obtains

$$
\begin{aligned}
(I I) & \leq\|\mu\|_{L^{\infty}(\mathbb{R})} \int_{0}^{T}\|\bar{W}(\tau)\|_{L^{2}(\Omega)}^{2} \mathrm{~d} \tau \\
& +C_{\mathrm{L}}\left\|B_{2}(\tau)\right\|_{L^{\infty}(\Omega \times(0, T))} \int_{0}^{T}\left(\frac{\|\bar{W}(\tau)\|_{L^{2}(\Omega)}^{2}}{2}+\frac{\|\bar{V}(\tau)\|_{L^{2}(\Omega)}^{2}}{2} \mathrm{~d} \tau .\right.
\end{aligned}
$$

Coming back to equation (30), it follows that

$$
\begin{aligned}
& \frac{1}{2}\|\bar{W}(T)\|_{L^{2}(\Omega)}^{2}+\int_{0}^{T} \int_{\Gamma_{\text {out }}} Q(x, \tau) \bar{W}(x, \tau)^{2} \mathrm{~d} x \mathrm{~d} \tau \\
& +\left(\lambda-\epsilon_{2}\|Q\|_{L^{\infty}(\bar{\Omega} \times(0, T))}-\|\mu\|_{L^{\infty}(\mathbb{R})}-\frac{C_{L}\left\|B_{2}\right\|_{L^{\infty}(\Omega \times(0, T))}}{2}\right) \int_{0}^{T}\|\bar{W}(\tau)\|_{L^{2}(\Omega)}^{2} \mathrm{~d} \tau \\
& +\left(D_{\mathrm{B}}-\frac{\|Q\|_{L^{\infty}(\bar{\Omega} \times(0, T))}}{4 \epsilon_{2}}\right) \int_{0}^{T}\|\nabla \bar{W}(\tau)\|_{L^{2}(\Omega)}^{2} \mathrm{~d} \tau \\
& \leq \frac{C_{L}\left\|B_{2}\right\|_{L^{\infty}(\Omega \times(0, T))}}{2} \int_{0}^{T}\|\bar{V}(\tau)\|_{L^{2}(\Omega)}^{2} \mathrm{~d} \tau .
\end{aligned}
$$

Finally, adding equations (29) and (31), we obtain

$$
\begin{aligned}
& \frac{1}{2}\left(\|\bar{V}(t)\|_{L^{2}(\Omega)}^{2}+\|\bar{W}(t)\|_{L^{2}(\Omega)}^{2}\right) \\
& +\left(\lambda-\epsilon_{1}\|Q\|_{L^{\infty}(\bar{\Omega} \times(0, T))}-\frac{\|\mu\|_{L^{\infty}(\mathbb{R})}}{2}-\frac{C_{L}\left\|B_{2}\right\|_{L^{\infty}(\Omega \times(0, T))}}{2}\right) \int_{0}^{T}\|\bar{V}(\tau)\|_{L^{2}(\Omega)}^{2} \mathrm{~d} \tau \\
& +\left(\lambda-\epsilon_{2}\|Q\|_{L^{\infty}(\bar{\Omega} \times(0, T))}-\frac{3}{2}\|\mu\|_{L^{\infty}(\mathbb{R})}-\frac{C_{L}\left\|B_{2}\right\|_{L^{\infty}(\Omega \times(0, T))}}{2}\right) \int_{0}^{T}\|\bar{W}(\tau)\|_{L^{2}(\Omega)}^{2} \mathrm{~d} \tau \\
& +\left(D_{\mathrm{B}}-\frac{\|Q\|_{L^{\infty}(\bar{\Omega} \times(0, T))}}{4 \epsilon_{2}}\right) \int_{0}^{T}\|\nabla \bar{W}(\tau)\|_{L^{2}(\Omega)}^{2} \mathrm{~d} \tau \\
& +\left(D_{\mathrm{S}}-\frac{\|Q\|_{L^{\infty}(\bar{\Omega} \times(0, T))}}{4 \epsilon_{1}}\right) \int_{0}^{T}\|\nabla \bar{V}(\tau)\|_{L^{2}(\Omega)}^{2} \mathrm{~d} \tau \leq 0 .
\end{aligned}
$$




$$
\begin{aligned}
\text { Choosing } \epsilon_{1}>\frac{\|Q\|_{L^{\infty}(\bar{\Omega} \times(0, T))}}{4 D_{\mathrm{S}}}, \epsilon_{2}>\frac{\|Q\|_{L^{\infty}(\bar{\Omega} \times(0, T))}}{4 D_{\mathrm{B}}} \text { and } \\
\qquad \lambda>\frac{C_{\mathrm{L}}\left\|B_{2}\right\|_{L^{\infty}(\Omega \times(0, T))}}{2}+\max \left\{\epsilon_{1}, \epsilon_{2}\right\}\|Q\|_{L^{\infty}(\bar{\Omega} \times(0, T))}+\frac{3}{2}\|\mu\|_{L^{\infty}(\mathbb{R})},
\end{aligned}
$$

it follows that $\|\bar{W}\|_{L^{2}\left(0, T, H^{1}(\Omega)\right)}+\|\bar{V}\|_{L^{2}\left(0, T, H^{1}(\Omega)\right)}=0$, which implies that $\bar{W}=\bar{V}=0$ in $\Omega \times(0, T)$.

Consequently $S_{1}=S_{2}$ and $B_{1}=B_{2}$ in $\Omega \times(0, T)$ and we have proved the statement of Theorem 3.9.

\section{Conclusion}

In this work, we have focused on the modeling of a continuous flow bioreactor in which a biomass and a substrate are interacting. We have carried out a mathematical analysis of the system of partial differential equations appearing in the model. We have stated the definition of solution and we have proved theoretical results showing the existence and uniqueness of solution under the assumptions of both linear and nonlinear reaction terms. We have also shown non-negativity and boundedness results for the solution. The results shown in this work are of interest for the study of this type of bioreactor models, their design and the optimization of the corresponding processes (see, e.g., [5, 11]).

\section{Acknowledgments}

This work was carried out thanks to the financial support of the Spanish "Ministry of Science and Innovation" under the project MTM2011-22658 and to the research group MOMAT (Ref.910480) supported by "Banco Santander" and "Universidad Complutense de Madrid"

\section{References}

[1] S.N. Antontsev, J.I. Díaz, and S. Shmarev. Energy Methods for Free Boundary Problems: Applications to Nonlinear Pdes and Fluid Mechanics. Progress in Nonlinear Differential Equations and Their Applications. Birkhäuser Boston, 2002.

[2] American Water Works Association. Water Treatment. Principles and practices of water supply operations series. American Water Works Association, 2003.

[3] J.E. Bailey and D.F. Ollis. Biochemical Engineering Fundamentals. McGraw-Hill Education, 1986.

[4] M. Ballyk, L. Dung, Donald Arthur Jones, and Hal Leonard Smith. Effects of random motility on microbial growth and competition in a flow reactor. SIAM J. Appl. Math., $59(2): 573-596,1998$.

[5] Juan Manuel Bello Rivas, Jérôme Harmand, Benjamin Ivorra, Angel Manuel Ramos, and Alain Rapaport. Bioreactor shape optimisation. modeling, simulation, and shape optimization of a simple bioreactor for water treatment. In 7th STIC \& Environnement 2011, pages 125-141. Transvalor - Presses des Mines, 2011.

[6] H. Brezis. Functional Analysis, Sobolev Spaces and Partial Differential Equations. Universitext. Springer, 2010. 
[7] A. K. Dram. A semilinear parabolic boundary-value problem in bioreactors theory. Electronic Journal of Differential Equations (EJDE) [electronic only], 2004, 2004.

[8] L.C. Evans. Partial Differential Equations. Graduate studies in mathematics. American Mathematical Society, 2010.

[9] A. Friedman. Partial Differential Equations of Parabolic Type. Prentice Hall Inc, 1964.

[10] I.M. Gelfand and G.E. Shilov. Generalized Functions: Properties and operations. Generalized Functions. Academic Press, 1964.

[11] Jrme Harmand, Alain Rapaport, and Alexandre Trofino. Optimal design of interconnected bioreactors: New results. AIChE Journal, 49(6):1433-1450, 2003.

[12] Cheng Ming Kung and Basil C. Baltzis. The growth of pure and simple microbial competitors in a moving distributed medium. Mathematical Biosciences, 111(2), 1992.

[13] O.A. Ladyzhenskaia, V.A. Solonnikov, and N.N. Ural'tseva. Linear and Quasi-linear Equations of Parabolic Type. American Mathematical Society, translations of mathematical monographs. American Mathematical Society, 1968.

[14] D. Lauffenburger, R. Aris, and K. Keller. Effects of random motility on growth of bacterial populations. Microbial Ecology, 7(3):207-227, 1981.

[15] D. Lauffenburger and B. Calcagno. Competition between two microbial populations in a nonmixed environment: Effect of cell random motility. Biotechnology and Bioengineering, 25(9):2103-2125, 1983.

[16] G.M. Lieberman. Intermediate schauder theory for second order parabolic equations. iv. time irregularity and regularity. Differential and Integral Equations, 5(6):1219-1236, 1992.

[17] Jacques Louis Lions. Sur les problèmes mixtes pour certains systèmes paraboliques dans les ouverts non cylindriques. Annales de l'institut Fourier, 7:143-182, 1957.

[18] Jacques Louis Lions. Contrôle optimal de systèmes governés par des équations aux dérivées partiales. 1968.

[19] Jacques Louis Lions and E. Magenes. Non-homogeneous Boundary value Problems and Applications. Volume I. 1972.

[20] Chia Ven Pao. Nonlinear Parabolic and Elliptic Equations. Fems Symposium. Plenum Press, 1992.

[21] Chia Ven Pao and Weihua Ruan. Positive solutions of quasilinear parabolic systems with nonlinear boundary conditions. Journal of Mathematical Analysis and Applications, 333(1):472 - 499, 2007.

[22] Zhipeng Qiu, Kaifa Wang, and Yun Zou. The asymptotic behavior of flowreactor models with two nutrients. Mathematical and Computer Modelling, 40(56):465 - 479, 2004.

[23] A.M. Ramos. Introducción al Análisis Matemático del Método de Elementos Finitos. Editorial Complutense, 2012.

[24] A. Rapaport, J. Harmand, and F. Mazenc. Coexistence in the design of a series of two chemostats. Nonlinear Analysis: Real World Applications, 9(3):1052 - 1067, 2008. 
[25] L. Shangerganesh and K. Balachandran. Existence and uniqueness of solutions of predator-prey type model with mixed boundary conditions. Acta Applicandae Mathematicae, 116(1):71-86, 2011.

[26] Hal Leonard Smith and P. Waltman. The theory of the Chemostat. In Cambridge studies in Mathematical biology, volume 13. Cambridge: Cambridge University Press, 1995.

[27] G. Teschl. Ordinary Differential Equations and Dynamical Systems. Graduate Studies in Mathematics. American Mathematical Society, 2012.

[28] C.Y. Wen and L.T. Fan. Models for Flow Systems and Chemical Reactors. Chemical Processing and Engineering. Dekker, 1975.

\section{A Classical existence and uniqueness results}

\section{A.1 Classical results regarding linear parabolic boundary value problems}

We consider $V, H$ Hilbert spaces with associated norms $\|\cdot\|$ and $|\cdot|, V \subset H$ and such that $V$ is dense on $H$. If we identify $V^{\prime}$ with the dual of $V$, it follows that

$$
V \subset H \subset V^{\prime} .
$$

Let us consider $\mathcal{V}=L^{2}(0, T, V)$ and $\mathcal{H}=L^{2}(0, T, H)$, where if $X$ is a Hilbert Space, $L^{2}(0, T, X)$ denotes the space of Lebesgue measurable functions $f:(0, T) \rightarrow X$ and such that

$$
\left(\int_{0}^{T}\|f(t)\|_{X}^{2} \mathrm{~d} t\right)^{\frac{1}{2}}<\infty .
$$

Then, it follows that $\mathcal{V}^{\prime}=L^{2}\left(0, T, V^{\prime}\right)$.

We also consider the space

$$
W\left(0, T, V, V^{\prime}\right)=\left\{u \mid u \in L^{2}(0, T, V), \frac{\mathrm{d} u}{\mathrm{~d} t} \in L^{2}\left(0, T, V^{\prime}\right)\right\}
$$

with the norm

$$
\|u\|_{W\left(0, T, V, V^{\prime}\right)}=\left(\int_{0}^{T}\|u(t)\|_{V^{2}}^{2} \mathrm{~d} t+\int_{0}^{T}\left\|\frac{\mathrm{d} u}{\mathrm{~d} t}(t)\right\|_{V^{\prime}}^{2} \mathrm{~d} t\right)^{\frac{1}{2}} .
$$

Theorem A.1 (Theorem 3.1 and Proposition 2.1 in [19]).

$$
W\left(0, T, V, V^{\prime}\right) \subset C^{0}([0, T], H) .
$$

Let $a(t, \cdot, \cdot)$ a bilinear and continuous form on $V$, a.e.t $\in(0, T)$, satisfying the following conditions:

$$
\left\{\begin{array}{l}
\forall u, v \in V \quad \text { the function } t \rightarrow a(t, u, v) \text { is measurable and } \\
\exists c \in \mathbb{R}:|a(t, u, v)| \leq c\|u\|\|v\| \quad \forall u, v \in V, \text { a.e.t } \in[0, T] .
\end{array}\right.
$$

There exists $\lambda, \alpha>0$ such that

$$
a(t, v, v)+\lambda|v|^{2} \geq \alpha\|v\|^{2} \quad \forall v \in V \text {, a.e.t } \in[0, T] .
$$

Since a.e. $t \in(0, T)$, the form $v \rightarrow a(t, u, v)$ is continuous on $V$, there exists $A(t) u \in V^{\prime}$ such that

$$
a(t, u, v)=<A(t) u, v>_{V^{\prime} \times V}
$$


which defines

$$
A(t) \in \mathcal{L}\left(V, V^{\prime}\right) .
$$

Let us consider the following evolution problem

$$
\left\{\begin{array}{l}
\text { Find } u \in W\left(0, T, V, V^{\prime}\right) \text { such that } \\
A(t) u+\frac{\mathrm{d} u}{\mathrm{~d} t}=f, \quad \text { where } f \in L^{2}\left(0, T, V^{\prime}\right), \\
y(0)=u_{0}, \quad \text { where } u_{0} \in H .
\end{array}\right.
$$

Definition A.2. We say that $u$ is a weak solution of (35), if it satisfies

$$
\frac{\mathrm{d}}{\mathrm{d} t}(u(\cdot), v)+a(u(\cdot), v)=<f(\cdot), v>_{V^{\prime} \times V} \quad \text { for all } v \in V,
$$

in the sense of $\mathcal{D}^{\prime}(] 0, T[)$, which means that the three terms of the equation above are to be considered as distributions in $t$.

Theorem A.3 (Theorem 1.2, Chapter III [18]). Assume Hypothesis (33) and (34) hold. Then Problem (35) has a unique weak solution.

\section{A.2 Fixed Point Theorem}

Theorem A.4 (Schauder Fixed Point Theorem: Theorem 3, Chapter IX 88). Assume $X$ is a Banach space, $K \subset X$ is compact and convex, and assume also

$$
A: K \longrightarrow K
$$

is continuous. Then, A has a fixed point in $K$.

\section{A.3 Embedding Theorems}

Definition A.5 (Compact operator). Assume $X, Y$ are Banach Spaces. We say that the operator $f: X \rightarrow Y$ is compact if given $U \subset X$ bounded, $f(U)$ is precompact in $Y$, i.e., $\overline{f(U)}$ is compact in $Y$.

Definition A.6 (Compact embedding). Assume $X, Y$ are Banach Spaces. We say that $X \subset$ $Y$ is compactly embedded if the identity operator id : $X \rightarrow Y$ is compact.

Lemma A.7 (Aubin-Lions Compactness Lemma). Let $X \subset B \subset Y$ Banach spaces such that the inclusion $X \subset B$ is a compact embedding. Then, for any $1<p<\infty, 1 \leq q \leq \infty$, the space

$$
\left\{f: f \in L^{p}(0, T, X) \text { and } \frac{\mathrm{d} f}{\mathrm{~d} t} \in L^{q}(0, T, Y)\right\}
$$

is compact embedded in $L^{p}(0, T, B)$.

Remark A.8. Particularly, if $q=p=2, X=H^{1}(\Omega)$ and $B=L^{2}(\Omega)$ and $Y=\left(H^{1}(\Omega)\right)^{\prime}$ it follows that

$$
W\left(0, T, H^{1}(\Omega),\left(H^{1}(\Omega)\right)^{\prime}\right) \subset L^{2}\left(0, T, L^{2}(\Omega),\right.
$$

with compact embedding. 


\section{A.4 Trace Theorem}

Theorem A.9 (Theorem 3.27, 23]). If $\Omega \subset \mathbb{R}^{n}$ is open and Lipschitz-regular and $\Gamma=\delta \Omega$, the trace operator $\gamma: H^{1}(\Omega) \rightarrow L^{2}(\Gamma)$ defined as $\gamma(u)=\left.u\right|_{\Gamma}$ is well defined and continuous, i.e., there exists a constant $C_{\mathrm{T}}>0$ such that

$$
\|\gamma(u)\|_{L^{2}(\Gamma)} \leq C_{\mathrm{T}}\|u\|_{H^{1}(\Omega)} .
$$

\section{A.5 Inequalities}

Proposition A.10 (Young's Inequality with $\epsilon>0[8]$ ).

$$
a b \leq \epsilon a^{2}+\frac{b^{2}}{4 \epsilon} \quad, \forall a, b \in \mathbb{R}, \epsilon>0 .
$$

Theorem A.11 (Gronwall's Inequality in its integral form [27]). Let $\varepsilon(\cdot), \chi(\cdot)$ and $\psi(\cdot)$ be real continuous functions defined on an interval $[a, b]$ and such that $\chi(t) \geq 0$, for $t$ in $[a, b]$, and

$$
\varepsilon(t) \leq \int_{a}^{t} \varepsilon(s) \chi(s) \mathrm{d} s+\psi(t) \quad \text { for all } t \in[a, b] .
$$

Then,

$$
\varepsilon(t) \leq \psi(t)+\int_{a}^{t} \chi(s) \psi(s) e^{e_{s}^{t} \psi(u) \mathrm{d} u} \mathrm{~d} s \quad \text { for all } t \in[a, b] .
$$

Furthermore, if $\psi$ is constant, then it follows

$$
\varepsilon(t) \leq \psi e^{\int_{a}^{t} \chi(s) \mathrm{d} s} \text { for all } t \in[a, b]
$$

\section{A.6 Functional Analysis}

Definition A.12. Let $X$ a Banach Space and $X^{\prime}$ its dual. We say that $\left(f_{\mathrm{n}}\right)_{n} \subset X^{\prime}$ converges to $f \in X^{\prime}$ in the weak-* topology, and we denote it by $f_{\mathrm{n}} \stackrel{*}{\rightarrow} f$, if

$$
<f_{\mathrm{n}}, x>_{X^{\prime} \times X} \stackrel{n \rightarrow \infty}{\longrightarrow}<f, x>_{X^{\prime} \times X}
$$

for every $x \in X$.

Theorem A.13 (Banach-Alouglu-Bourbaki (Theorem 3.16, [6]). Let $X$ be a Banach space and $X^{\prime}$ its dual. The unit ball

$$
B_{X^{\prime}}=\left\{f \in X^{\prime} \mid\|f\|_{X^{\prime}} \leq 1\right\}
$$

is compact in the weak-* topology.

Theorem A.14 (Theorem 3.18, [6]). Let $X$ a separable space and $\left\{f_{\mathrm{n}}\right\}_{n} \subset X^{\prime}$ a bounded sequence, then there exists a subsequence $\left\{f_{\mathrm{n}_{\mathrm{k}}}\right\}_{k}$ that converges in the weak-* topology to some $f \in X^{\prime}$.

Theorem A.15 (Theorem 4.9, [6]). Let $1 \leq p \leq \infty$. If $\left\{f_{\mathrm{n}}\right\}_{n} \subset L^{p}(Q)$ and $f \in L^{p}(Q)$, such that $\left\|f_{\mathrm{n}}-f\right\|_{L^{p}(Q)} \rightarrow 0$, then there exists a subsequence $\left\{f_{\mathrm{n}_{\mathrm{k}}}\right\}_{k}$ such that $f_{\mathrm{n}_{\mathrm{k}}} \rightarrow f$ almost everywhere in $Q$.

Theorem A.16 (Proposition 3.13(iv), [6]). If $\left\{f_{\mathrm{n}}\right\}_{n} \subset X^{\prime}$ converges to $f \in X^{\prime}$ in the weak-* topology, $\left\{x_{\mathrm{n}}\right\}_{n} \subset X, x \in X$ such that $\left\|x_{\mathrm{n}}-x\right\|_{X} \rightarrow 0$, then

$$
<f_{\mathrm{n}}, x_{\mathrm{n}}>_{X^{\prime} \times X} \rightarrow<f, x>_{X^{\prime} \times X}
$$

